\title{
Circalunar variations of the night sky brightness - an FFT perspective on the impact of light pollution
}

\author{
Johannes Puschnig, ${ }^{1, *}$ Stefan Wallner, ${ }^{2,3}$ Thomas Posch ${ }^{2}$ \\ * e-mail: johannes.puschnig@uni-bonn.de
}

1 Universität Bonn, Argelander-Institut für Astronomie, Auf dem Hügel 71, D-53121 Bonn, Germany

2 Universität Wien, Institut für Astrophysik, Türkenschanzstraße 17, A-1180 Wien, Austria

3 ICA, Slovak Academy of Sciences, Dubravska cesta 9, 84503 Bratislava, Slovak Republic

Accepted for publication in MNRAS on Dec 11, 2019.

\begin{abstract}
Circa-monthly activity conducted by moonlight is observed in many species on Earth. Given the vast amount of artificial light at night (ALAN) that pollutes large areas around the globe, the synchronization to the circalunar cycle is often strongly perturbed. Using two-year data from a network of 23 photometers (Sky Quality Meters; SQM) in Austria (latitude $\sim 48^{\circ}$ ), we quantify how light pollution impacts the recognition of the circalunar periodicity. We do so via frequency analysis of nightly mean sky brightnesses using Fast Fourier Transforms. A very tight linear relation between the mean zenithal night sky brightness (NSB) given in magSQM $\operatorname{arcsec}^{-2}$ and the amplitude of the circalunar signal is found, indicating that for sites with a mean zenithal NSB brighter than 16.5 magSQM $\operatorname{arcsec}^{-2}$ the lunar rhythm practically vanishes. This finding implies that the circalunar rhythm is still detectable (within the broad bandpass of the SQM) at most places around the globe, but its amplitude against the light polluted sky is strongly reduced. We find that the circalunar contrast in zenith is reduced compared to ALAN-free sites by factors of $1 / 9$ in the state capital of Linz ( 200,000 inhabitants) and 1/3 in small towns, e.g. Freistadt and Mattighofen, with less than 10,000 inhabitants. Only two of our sites, both situated in national parks (Bodinggraben and Zöblboden), show natural circalunar amplitudes. At our urban sites we further detect a strong seasonal signal that is linked to the amplification of anthropogenic skyglow during the winter months due to climatological conditions.
\end{abstract}

\section{Introduction}

\subsection{Impact of moonlight on animals, plants and humans}

The Moon's synodic period of 29.5 days is its orbital time around the Earth required to show the exact same lunar phase, i.e. for example the time span between two consecutive full moons. The corresponding circalunar oscillation of the Moon's illumination impacts many types of life on Earth, in particular in the context of reproduction cycles. Scientific work on this topic dates back to the early $20^{\text {th }}$ century (e.g. Fox and Gardiner (1924)) and it was likely already recognized by fishermen in the antiquity - due to practical implications - that the size of (edible) gonads of sea urchins varies over the lunar month (Raible et al., 2017). 
Later studies revealed that also predator-prey interactions change with moon phase and illumination (Clarke, 1983; Shimose et al., 2013), giving advantages to either side, depending on the context. More recently, Fallows et al. (2016) studied interactions between white sharks (Carcharodon carcharias) and Cape fur seals (Arctocephalus pusillus pusillus). They found that the shark attack frequency and seal capture success was significantly higher at sunrise during periods of low lunar illumination. In other species, the lunar cycle may control activity (Kolb, 1992), foraging, habitat use and communication. See Kronfeld-Schor et al. (2013) for a recent review on those topics. And for the golden rabbitfish (Siganus guttatus), Takemura et al. (2006) found a direct influence of moonlight intensity on changes in melatonin production.

Some animals are driven by the Moon in their orientation (Papi and Pardi, 1963; Frisch, 1993), in particular Dacke et al. (2011) found that dung beetles (carabaeus lamarcki) use the polarization pattern around the Moon as a compass for maintaining their travel direction.

Bünning and Moser (1969) studied how different types of plants react on moonlight. They revealed that plants may undergo leaf movements such that the intensity of lunar illumination is reduced and disturbing effects caused by moonlight are eliminated. It was further shown that illumination by moonlight may even promote flowering, e.g. Ben-Attia et al. (2016) found flowering patterns in the cactus Cereus peruvianus with a period of $\sim 29.5$ days and a correlation between moon phase and number and proportion of flowers in bloom. In aquatic systems, Zantke et al. (2013) first established that the marine worm Platynereis dumerilii possesses an endogenous circalunar clock and Last et al. (2016) was one of the first to search for impacts on aquatic ecosystems and especially noticed a vertical migration of zooplankton which takes place in winter when the Moon is above the horizon at the Arctic, fjord or other sea areas.

Besides animals and plants, the impact of moonlight on humans is still under debate, see for example Zimecki (2006) for a review. However, many authors find evidence that women of reproductive age do follow the circalunar rhythm (Reinberg et al., 2016), especially the ovulation seems to accumulate around new moon (Law, 1986).

What about the effect of lunar illumination on human sleep? On one hand, Cajochen et al. (2013) find that at full moon the electroencephalogram delta activity during the deep sleep phase is 30 percent decreased and that the sleep duration is reduced by 20 minutes, but on the other hand, Cordi et al. (2014) find no such significant effects depending on lunar cycle. However, Cajochen et al. (2014) pointed out that the volunteers tested by Cordi et al. (2014) were not synchronised with respect to their own natural sleep timing, which may have led to low signal-to-noise in their result.

Given the plethora of studies about how moonlight impacts various species on Earth, our work on how artificial light at night (ALAN) impacts the recognizability of the lunar cycle, seems to be a timely matter, as ALAN thus presumably perturbs those species in manifold ways (reproduction, predator-prey interaction, activity, orientation, and so forth) as well.

\subsection{Moonlight versus light pollution}

Given the fact that ALAN is ever increasing on a global scale (Kyba et al., 2015), effects of artificial light on organisms and ecosystems have gained increasingly more attention in recent years (Hölker et al., 2010; Gaston et al., 2015; Manfrin et al., 2017). It has become clear that ALAN indeed has multifaceted consequences for flora and fauna, see e.g. Schroer and Hölker (2016) for a review.

However, despite the fact that the influence of moonlight on beings on our planet is well documented (see previous section), and that ALAN's impact on various organisms and ecosystems have been demonstrated by many authors, to date no studies were performed to investigate ALAN's impact on processes that rely on synchronization with moonlight. The reason for this knowledge gap is that the community is lacking a quantification of the strength of the circalunar rhythm in dependence of the level of light pollution (Davies et al., 2013). With this work, we aim to provide such a quantification, i.e. a simple empirical relation between the mean night sky brightness and the 




Figure 1. Skycalc radiance model for the zenith with the full moon at 45 degree altitude. Transmission curves of the SQM and Bessel B, V, R filters are overplotted.

amplitude of the circalunar rhythm, allowing the knowledge gap to be filled in the near future.

\section{Locations and methods of our measurements}

The present paper is primarily based on zenithal night sky brightness (NSB) measurements taken with Sky Quality Meters (model SQM-LE). These are photodiode based devices with an optical element on the front that narrows down the field of view to a Gaussian-like cone with a full-with-at-half-maximum of $\sim 20^{\circ}$. Its effective bandpass ranges from approximately $400-650 \mathrm{~nm}$ (see Figure 1). Technical characterisation and testing was done by Cinzano (2005) and details about the absolute radiometric calibration are found in Bará et al. (2019).

Our SQM measurements have been carried out in the Austrian county of Upper Austria at 23 sites, distributed over the whole area of this county and ranging from its capital city Linz - which has very bright skies - to very remote locations such as Krippenstein on the Dachstein plateau $(\sim 2000 \mathrm{~m}$ above sea level). See Table 1 for station codes and geographic coordinates. Measurements are taken in an automated way, with SQM devices located in weather-proof housings. The network of SQMs is run by the provincial government of Upper Austria. Starting with six devices in 2014, it has grown to 23 SQMs by 2016. A detailed description of the individual sites, their exact locations and light pollution levels are found in Posch et al. (2018).

Measurements are taken every minute, thus a huge amount of data is generated every night. However, the present data analysis is based on the mean nocturnal NSBs $(<\mathrm{NSB}>)$ and is restricted to data obtained during the years 2016-2017. For each night and SQM site, we calculate $<$ NSB $>$ as arithmetic means of the minute-by-minute SQM readings for each night. The data series is further constrained for solar elevations below -15 degrees, in the same way as described by Posch et al. (2018). The contribution of scattered sunlight to the night sky brightness is negligible below this altitude. Our $<\mathrm{NSB}>$ measurements range from 17.3-21.0 magSQM $\operatorname{arcsec}^{-2}$.

We stress that we do not apply any further filtering, i.e. data obtained when the sky was cloudy are included.

\section{Synthetic models of ground illumination by the Moon and total zenithal night sky brightness}

In order to study ALAN's impact on the lunar rhythm, we first want to understand the amplitude of the naturally occuring oscillation of moonlight at our sites, i.e. without any contribution of anthropogenic light at night. We do so using two models. The first one, describing the lunar 
Table 1. Basic information of the 23 SQM locations in Upper Austria, categorized into urban, intermediate and rural sites, as done in Posch et al. (2018).

Code Name Latitude N Longitude E Elevation [m] (above sea level)

\begin{tabular}{|c|c|c|c|c|}
\hline & & & "urban & \\
\hline LSM & Linz, Schlossmuseum & N 481819 & E 141658 & 287 \\
\hline LGO & Linz, Göthestraße & N 481819 & E 141830 & 259 \\
\hline LSW & Linz, Sternwarte & N 481736 & E 14166 & 336 \\
\hline STY & Steyr & N 48257 & E 142632 & 307 \\
\hline STW & Steyregg-Weih & N 481719 & E 142113 & 331 \\
\hline TRA & Traun & N 48148 & E 141511 & 269 \\
\hline WEL & Wels, Rathaus & N 48923 & $\begin{array}{l}\text { E } 14129 \\
\text { intermediate }\end{array}$ & 317 \\
\hline $\mathrm{BRA}$ & Braunau & N 481540 & E 13241 & 351 \\
\hline GRI & Grieskirchen & N 48144 & E 134933 & 336 \\
\hline FRE & Freistadt & N 483033 & E 14307 & 512 \\
\hline MAT & Mattighofen & N 48550 & E 1396 & 454 \\
\hline PAS & Pasching & N 481531 & E 141236 & 292 \\
\hline VOE & Vöcklabruck & N 48021 & $\begin{array}{l}\text { E } 133843 \\
\text { rural }\end{array}$ & 434 \\
\hline BOD & Nationalpark Bodinggraben & N 474731 & E 142338 & 641 \\
\hline FEU & Feuerkogel & N 474857 & E 134315 & 1628 \\
\hline GIS & Giselawarte & N 48233 & E 141511 & 902 \\
\hline GRU & Grünbach & N 483150 & E 143430 & 918 \\
\hline KID & Kirchschlag-Davidschlag & N 482631 & E 141626 & 813 \\
\hline KRI & Krippenstein & N 473123 & E 134136 & 2067 \\
\hline LOS & Losenstein, Hohe Dirn & N 475422 & E 142440 & 982 \\
\hline MUN & Münzkirchen & N 482845 & E 133329 & 486 \\
\hline ULI & Ulrichsberg, Schöneben & N 484220 & E 135644 & 935 \\
\hline $\mathrm{ZOE}$ & Nationalpark Zöbloden & N 475018 & E 142628 & 899 \\
\hline
\end{tabular}


Table 2. Resulting values of the theoretical Moon model for half and full moons. ALT is the lunar altitude, PA is the lunar phase angle and $\mathrm{G}_{\mathrm{ILL}}$ is the ground illumination caused only by moonlight.

\begin{tabular}{ccc} 
ALT $\left[^{\circ}\right]$ & PA $\left[{ }^{\circ}\right]$ & GILL $_{\text {mmlux}]}$ \\
\hline \hline 0 & 90 & 0.192 \\
0 & 180 & 1.622 \\
45 & 90 & 19.474 \\
45 & 180 & 164.059 \\
90 & 90 & 31.949 \\
90 & 180 & 269.153
\end{tabular}

variation of ground illumination in units of lux and the second one, describing the naturally occuring variation of zenithal night sky brightness in units of magSQM $\operatorname{arcsec}^{-2}$, which may be approximately converted to luminance in units of $\mathrm{cd} \mathrm{m}^{-2}$ (Bará, 2016, 2017), using Equation 1. Despite the fact that this formula is widely used to estimate luminances from SQM magnitudes, it was originally derived by Garstang (1986) for Johnson V-band magnitudes, and is thus just an approximation.

$$
\text { Luminance }\left[\mathrm{cd} / \mathrm{m}^{2}\right]=10.8 \times 10^{4} \times 10^{\left(-0.4 \times\left[\mathrm{mag} \mathrm{arcsec}^{-2}\right]\right)}
$$

\subsection{Simplified model of ground illumination by the Moon}

\subsubsection{General solution for all possible values}

To obtain insight into the contribution of moonlight to the total ground illumination, we make use of the moonlight model by Seidelmann (1992). The model does not take into account contributions of the skyglow, stars or airglow and thus depend only on two parameters: the mean altitude of the Moon $\left(0-90^{\circ}\right.$, where $90^{\circ}$ is the zenith) and the Moon's phase angle $\left(0-180^{\circ}\right.$, where $180^{\circ}$ is full moon). Note that the parallax value is neglected due to its very small contribution. We show the whole parameter space covered by the model in Figure 2 .

Unsurprisingly, the amount of ground illuminance is highest when the full moon can be observed in the zenith. It is recognized that with increasing phase angle and altitude the illumination does not increase linear, but rather exponential, owed to the fact that the transmittance of the atmosphere is proportional to $e^{-\frac{\tau}{\cos (z)}}$, with $\tau$ being the optical depth and $z$ the zenith distance. Some values which underline this can be found in Table 2. In fact, for zenithal positions, there is a factor $\sim 8.4$ in ground illumination between full and half moon. The large difference is caused by coherent backscattering or opposition surge (Hapke et al. 1998). In this phenomenon portions of waves traveling along same paths but in opposite directions, interfere constructively with each other, causing a peak at zero phase (full moon).

In case of full moon the illuminance varies by a factor of $\sim 166$ between zenithal and horizontal positions. Please note that horizontal position, i.e. altitude of zero degrees, is valid for the center of the lunar disk. Hence, a slight ground illumination is visible and not zero. The resulting values can be considered as theoretical only, since every other possible light emitting source is neglected, even stars or the Milky Way are not taken into account. However, the model shows how the lunar position and its phase angle are associated with the consequential ground illumination.

\subsubsection{Application of the illuminance model to one of our sites}

We now apply the model to one of our sites, namely VOE. To do so, we first calculate for the years 2016-2017 the altitude and phase of the Moon at midnight and then derive the corresponding illuminance through the model. The result is shown in Figure 3. A strong seasonal variation is recognized in the model, mainly caused by the changing altitude of the ecliptic (and thus the Moon) 


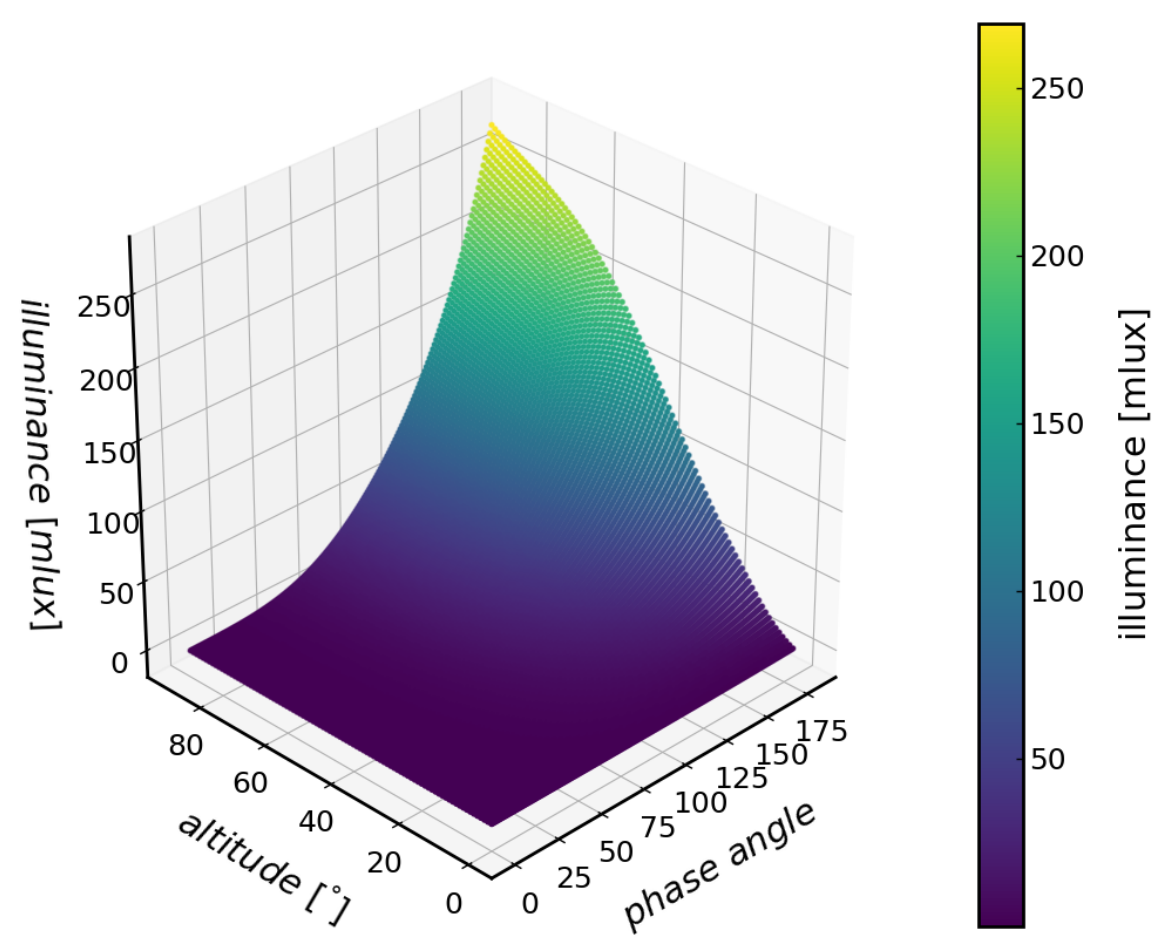

Figure 2. Results of the illuminance model as developed by Seidelmann (1992) for all phase angles and altitudes of the Moon.

between summer and winter, leading to a natural variation of the moon illuminance by a factor of $\sim 3.6$ (peak-to-peak for full moon).

\subsection{Zenithal night sky model for the SQM band}

\subsubsection{The Cerro Paranal Advanced Sky Model}

Using the Cerro Paranal Advanced Sky Model (Skycalc), we are able to compare our zenithal SQM measurements to a synthetic sky model that is cloud-free and takes into account several sources of light such as scattered moonlight, starlight, molecular emission of the lower atmosphere and the airglow (upper atmosphere).

Skycalc was published by Noll et al. (2012) and Jones et al. (2013), as part of an Austrian in-kind contribution to the European Southern Observatory (ESO), e.g. ESO's exposure time calculator is based on it. Skycalc's current version (2.0.4) also comes with a Python-based command line interface 1 . However, the model used for our study is based on Skycalc 1.4.4, which was available through a web interface only. The main input parameters are zenith distance (or airmass) of the observation, precipitable water vapor (PWV) and monthly averaged solar flux. For the moon radiance component, the separation of Sun and Moon as seen from Earth, the Moon-target separation, moon altitude over horizon and the Moon-Earth distance are needed.

We have decided to make some simplifications, allowing us to evaluate the model on a 2-dimensional parameter grid with vectors of (Sun-Moon-separation, moon altitude) only. This is reasonable in our case, because the measurement devices we are using, the Sky Quality Meters of type SQM-LE, are equipped with a front lens that narrows down the field of view to a roughly 20 degree wide cone, pointed towards zenith. Hence, we only need to consider zenithal night sky brightness. The two input parameters Moon-target separation and moon altitude can thus be

1/https://www.eso.org/observing/etc/doc/skycalc/helpskycalccli.html 


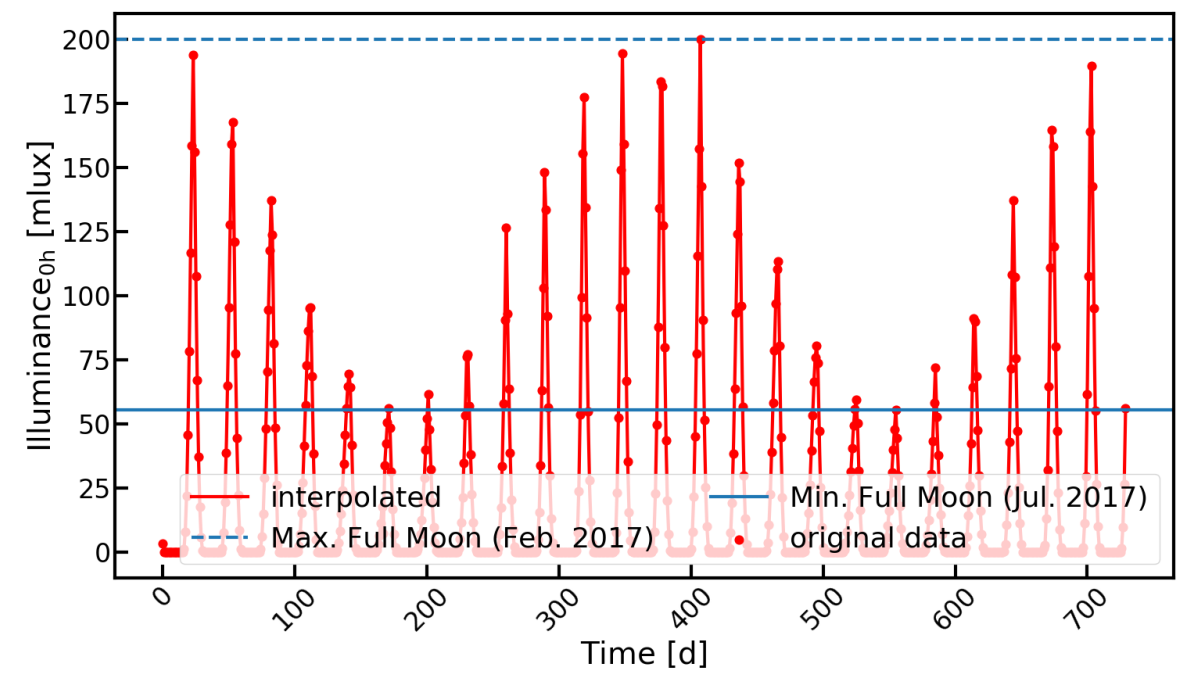

Figure 3. Ground illuminance (the Moon's contribution to it) evaluated for the location of Vöcklabruck, Upper Austria, over the years 2016-2017. The high-frequency lunar cycle is modulated with a seasonal variation caused by the seasonal change of the Moon's altitude.

simplified to one parameter, with the former one being the moon zenith distance. We have further decided to evaluate the model for a fixed PWV value of 5mm, a monthly averaged solar flux of 130sfu and for a fixed mean Moon-Earth distance. These simplifications have practically no influence on our results, since ALAN's contribution to our SQM measurements is magnitudes larger than the natural variation caused by phenomena such as PWV or solar flux. However, variations due to moon phase and height are fully treated by our gridded model evaluation for the zenith. Since the natural, cloudless sky brightness changes smoothly, a grid spacing of one degree in both parameters (Sun-Moon-separation, moon altitude) was found to be sufficient.

The result is a synthetic (cloud-free) night sky spectrum for the target location in units of photons $\mathrm{s}^{-1} \mathrm{~m}^{-2} \mu \mathrm{m}^{-1} \operatorname{arcsec}^{-2}$, i.e. spectral radiance. We first convert to erg s${ }^{-1} \mathrm{~cm}^{-2} \AA^{-1} \operatorname{arcsec}^{-2}$ and then multiply with the SQM transmission curve as published in Cinzano (2005) and shown in Figure 1, together with transmission curves of Bessel BVR filters. The radiance within the SQM band is then calculated via integration over the wavelength axis. Using a zeropoint (ZP) of -12.92 (Puschnig et al. in prep), we finally convert to magSQM $\operatorname{arcsec}^{-2}$ via equation 2 .

$$
N S B\left[\operatorname{mag}_{S Q M} \operatorname{arcsec}^{-2}\right]=-2.5 \times \log _{10}\left(\text { radiance }\left[\operatorname{erg~s}^{-1} \mathrm{~cm}^{-2} \operatorname{arcsec}^{-2}\right]\right)+Z P
$$

Note that Equation 2 results to a modeled zenithal sky brightness of $21.87 \mathrm{mag}_{\mathrm{SQM}} \operatorname{arcsec}^{-2}$ for new moon, which is in agreement with SQM observations of remote, rural sites (Posch et al., 2018; Bertolo et al. 2019, Bará et al., 2019). We further stress that the exact absolute value is not critical for the scientific results of the paper.

\subsubsection{Application of the Cerro Paranal Advanced Sky Model to one of our sites}

Analogous to Section 3.1.2, we now evaluate the Skycalc model for one of our sites (VOE) that we use as a proxy for our network. We do so for the years 2016-2017. However, in this case we calculate for each night the mean NSB $(<\mathrm{NSB}>)$ rather than just the value at midnight. The result is shown in Figure 4. As for the illuminance model, a strong seasonal variation is recognized, that is mainly caused by the changing altitude of the Moon. However, the relative change between brightest and darkest $<\mathrm{NSB}>\left(18.04\right.$ vs. 18.69 magSQM $_{\text {arcsec }}{ }^{-2}$ ) corresponds to a (linear-scale) factor of 1.8 only, which is half the amplitude that is seen in the illuminance model. The main reason for this 


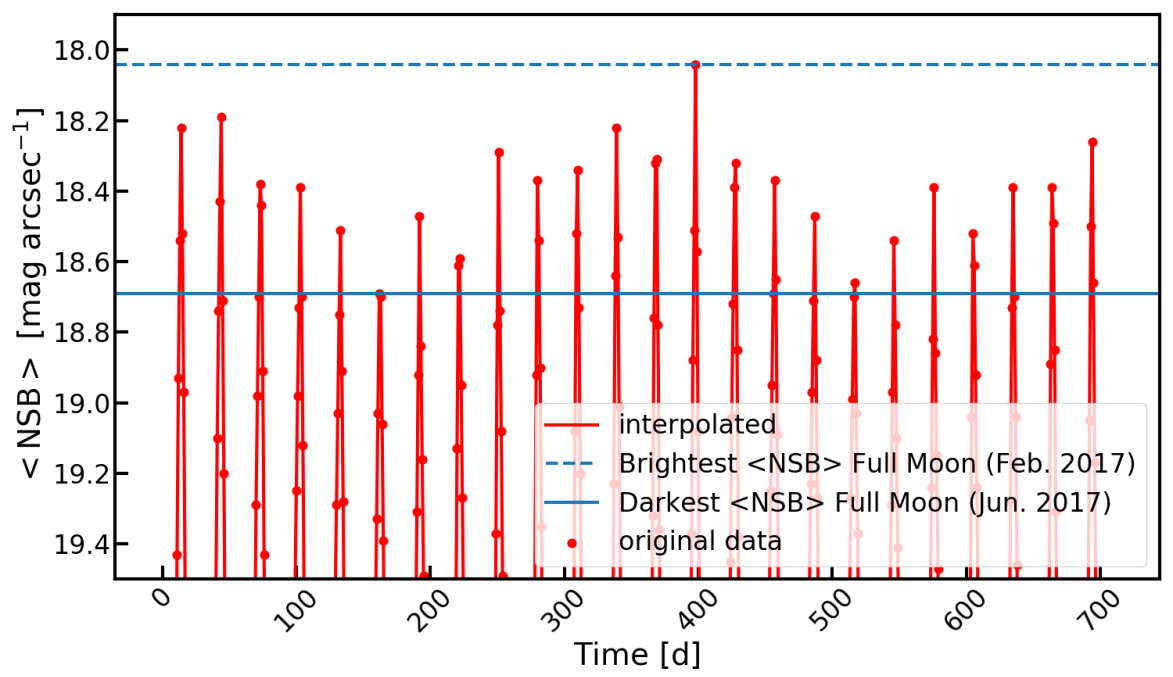

Figure 4. Skycalc sky model for the zenith, evaluated for one of our sites (VOE) for the years 20162017. The y-axis was limited to show only the bright peaks around full moon. Beside the circa-monthly oscillation, a seasonal variation is recognised, caused by changing altitude of the Moon between summer and winter.

discrepancy lies in the fact that we evaluate the illuminance model at midnight only, i.e. at the point of maximum illumination, whereas for Skycalc we calculate the nightly means within dark-time limits. Given the fact that summer nights are shorter, the relative contribution of the full moon to the zenithal $<\mathrm{NSB}>$ is thus higher in summer than in winter and the dynamic range of the seasonal variation shrinks in that case.

\section{Data analysis}

\subsection{Fourier analysis}

Using our 2-year data of nightly mean $<\mathrm{NSB}>$ values, we aim to reveal the amplitude of the circalunar periodicity (and other periodic signals that might exist). Numerous implementations of discrete Fourier transforms exist, but probably the most common one is the Fast Fourier Transform (FFT), which we also use for the analysis of our SQM data. In particular, we apply the FFT algorithm as implemented in numpy (Van Der Walt et al., 2011), the fundamental package for scientific computing with Python. In the following, we describe some general properties of the FFT and highlight common obstacles of the method and how we treat them.

\subsubsection{Which unit to choose?}

The SQM delivers data in units of magSQM $\operatorname{arcsec}^{-2}$. However, conversions into linear units are available, see Equation 1. Thus, we investigate how well the amplitude of periodic variations such as the circalunar cycle caused by varying moon phases or the seasonal rhythm, that is driven by variations of the moon zenith distances between summer and winter, are recovered in frequency space after application of the FFT in dependence of the data input unit.

For a linear input, the increase in zenithal night sky brightness from new moon to full moon starts very shallow, almost unrecognizable. Then, few days before full moon, the increase accelerates to finally form a sharp peak in the (time, $<\mathrm{NSB}>$ ) plane (see Figure 13). On the contrary, in logarithmic units such as magSQM $\operatorname{arcsec}^{-2}$ the phase of the shallow increase is more pronounced 
(stretched in time) and the later steep increase somewhat compressed. Thus, the apparent course in the (time, $<\mathrm{NSB}>$ ) plane is rather smooth compared to linear units (see Figure 12 ).

Since the FFT of a signal that is spread out in time delivers a compact result in frequency space and vice versa, logarithmic units such as magSQM $\operatorname{arcsec}^{-2}$ are preferred, because the smoother course leads to a better definition of the circalunar cycle in frequency space, i.e. a single peak.

\subsubsection{The role of gaps in $<\mathrm{NSB}>$ measurements}

Gaps in SQM data series can occur due to several reasons, be it hardware- or software-failures or even meteorological conditions. In this section, we investigate how gaps in the time series affect the ability of the FFT algorithm to correctly reproduce amplitudes of time-dependent variations. We do so by introducing single and double gaps of varying lengths into our synthetic sky model. The recovered amplitudes of the circalunar and seasonal rhythm are then evaluated against the gap fraction, i.e. the fraction of data points on the equal-distant input time grid without measurement.

We stress that under all circumstances gaps should not be left as such, but replaced with some form of interpolation or reasonable value. We made tests using linear, quadratic and cubic spline interpolations, as well as using the mean of the remaining data as fill value. We find that the latter one is the most robust and preferred method. Although cubic spline interpolation gives slightly better results for small gaps, i.e. when the gaps size is much smaller than the periodicity of the desired signal, it can cause unforeseen results for larger gaps.

Our results are summarized in table 3, which shows that the single- and double gap tests give very similar results: gap fractions of $1 \%, 5 \%, 7.5 \%$ and $10 \%$ recover the amplitude of the circalunar variation (A) at levels of 99\%, 92-95\%, 91\% and 87-88\% respectively. Even gap fractions of $20 \%$ recover $75-77 \%$ of the true amplitude.

The seasonal variation (S) is less affected, because of its longer periodic time. It can be accurately derived even for gap fractions of $20 \%$.

However, as described by Munteanu et al. (2016), for even larger periods of missing data, one should consider to perform spectral analysis using other algorithms such as the $Z$ transform or the Lomb-Scargle algorithm. They might be able to reproduce the amplitude for cases where gaps make up more than 50 percent of the time series.

Additionally, the presence of gaps in the time series leads to an increase of 'frequency noise' in the amplitude spectrum, limiting the chance to detect low-amplitude variations at certain frequencies. As shown in Table 3 , the noise roughly doubles between 0 and $5 \%$ gap fractions, but stays almost constant from thereon.

\subsubsection{Importance of the length of the data series for FFTs}

The length of a time series as well as its sampling rate are of importance for FFT studies, because they define the frequency resolution in the final amplitude spectrum.

Our sampling rate $f_{s}$ is one measurement per night or $f_{s}=1 d^{-1}$. The distance $\Delta$ t between two data points is: $\Delta t=\frac{1}{f_{s}}=1 \mathrm{~d}$. The final frequency range is thus limited to the interval $\left[-\frac{f_{s}}{2} ;+\frac{f_{s}}{2}\right]$, i.e. $-0.5 \ldots 0.5 \mathrm{~d}^{-1}$. The highest measurable frequency is $0.5 \mathrm{~d}^{-1}$ or two days periodic time. The number of discrete points $\mathrm{N}$ in the final frequency domain equals the number in time domain. Thus, the distance $\Delta^{\circ}$ in frequency space is $\Delta^{\circ}=\frac{\mathrm{f}_{\mathrm{s}}}{\mathrm{N}}$, which shows that the frequency resolution is controlled by the sampling rate $f_{s}$ and the length of the time series N. Since our sampling rate is fixed, the number of data points is the main quantity that drives the frequency resolution in our final amplitude spectrum.

For example, if we wanted to detect the frequency of the lunar synodic month (without prior knowledge), with an accuracy of $0.5 \mathrm{~d}$, we would need at least 1711 data points, equivalent to 4.68 years at a sampling rate of one measurement per night.

However, with prior knowledge of the period - in our case $29.5 \mathrm{~d}$ for the synodic month - one can adjust the time axis such that the final discrete amplitude spectrum covers the corresponding 
Table 3. We test the influence of single and double gaps on the ability to recover signal amplitudes from synthetic sky model data. The gap fraction in percent is given in column 1 and the recovered amplitudes of the circalunar and the seasonal variation are shown in column 2 and 3 for the single gap case, and column 5 and 6 for the double gap test. FFT Noise is given in column 4 and $\%$. The unit for all measurements is magSQM $\operatorname{arcsec}^{-2}$.

\begin{tabular}{lcccccc} 
& \multicolumn{3}{c}{ single gap } & \multicolumn{3}{c}{ double gap } \\
GF & $\mathrm{A}$ & $\mathrm{S}$ & $\mathrm{N}$ & $\mathrm{A}$ & $\mathrm{S}$ & $\mathrm{N}$ \\
$(1)$ & $(2)$ & $(3)$ & $(4)$ & $(5)$ & $(6)$ & $(7)$ \\
\hline \hline 0 & 1.508 & 0.073 & 0.006 & 1.508 & 0.073 & 0.006 \\
1 & 1.489 & 0.077 & 0.008 & 1.488 & 0.075 & 0.009 \\
5 & 1.415 & 0.078 & 0.013 & 1.438 & 0.083 & 0.011 \\
7.5 & 1.397 & 0.070 & 0.012 & 1.403 & 0.078 & 0.014 \\
10 & 1.356 & 0.081 & 0.012 & 1.349 & 0.075 & 0.015 \\
20 & 1.217 & 0.072 & 0.014 & 1.205 & 0.074 & 0.015 \\
30 & 1.037 & 0.060 & 0.015 & 1.067 & 0.067 & 0.015 \\
50 & 0.754 & 0.036 & 0.013 & 0.765 & 0.010 & 0.015
\end{tabular}

frequency, i.e. $\frac{1}{29.5}=0.0339 \mathrm{~d}^{-1}$. This is achieved by limiting the data points such that no discontinuities occur at the edges of the time series, i.e. spectral leakage (see next paragraph) is eliminated. That way, even time series of only one year recover the amplitude on levels better than 90 percent.

\subsubsection{Avoiding spectral leakage}

The ability of the FFT algorithm (and any other discrete Fourier transforms) to recover amplitudes is limited due to the fact that the duration of the observation is finite. This means that the input signal is factual a product with a rectangular window. The discrete spectrum of any finite signal is thus spread out over multiple frequency components and the amplitude is not fully recovered anymore. This behaviour is called spectral leakage.

However, the effect may be reduced by 1) avoiding discontinuities of the input signal or 2) gradually decreasing the amplitude of the signal towards the edges of the measurement series. The first method requires prior knowledge of the periodic time and phase of the signal of interest (which in most applications is not fulfilled). The latter case can be achieved by multiplying the input time series with a window function, e.g. a Hanning window, before the FFT is applied.

Since the periodicity of the synodic month is known, we could test both scenarios using sinusoidal and our synthetic sky time series as input. Although the application of a Hanning window improved amplitude recovery in cases where discontinuities occurred at the edges, we find that an continuous input time series (e.g. from first new moon 2016 to last new moon in 2017) gives best results with only negligible amounts of spectral leakage and amplitude recovery at levels better than 95 percent. Thus, for our main science case, i.e. studying the circalunar rhythm, a continuous time series as input for the FFT is recommended and all our FFT analysis was performed using data from new moon 2016/01/09 to new moon 2017/12/18 only.

\section{Results}

\subsection{FFT analysis of the illuminance and Skycalc models}

As a reference for our measurement sites, we perform an FFT analysis of our model data using the considerations from Section 4.1. To do so, for the Skycalc model we first calculate the nightly 


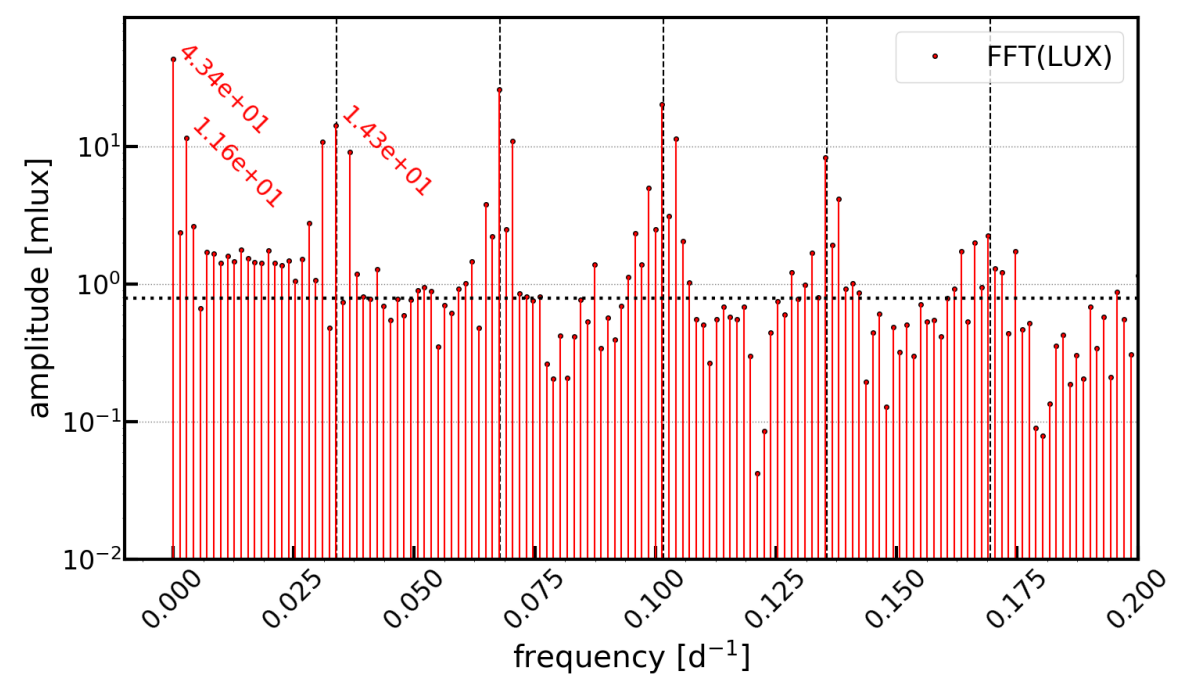

Figure 5. FFT amplitude spectrum of the illuminance model as described in Section 3.1. The shown frequency range is limited to values between 0 and 0.2 . Note that the unit of the y-axis is milli-lux. Labeled amplitudes (from left to right correspond to the mean of all input data, the seasonal variation and the circalunar cycle.

averages, i.e. $\langle\mathrm{NSB}>$, as described in Section 2, The resulting FFT amplitude spectra are shown in Figures 5 and 6 .

An analysis of both models reveals significantly strong amplitudes at the frequency of $\frac{1}{29.5}=0.0339 \mathrm{~d}^{-1}$, i.e. the fundamental of the circalunar rhythm. Also its harmonics are identified at multiples of that frequency. However, the noise level in the illuminance model is higher because of its peak-like input (see Section 4.1.1).

We also note that the zero-frequency amplitude is not comparable between the models. While for the Skycalc model, the zero-frequency is in fact a representation of the mean $\langle\mathrm{NSB}\rangle$, this is not true for the illuminance model, because for the FFT analysis all input data with illuminance levels of olux is considered as gaps and are thus filled with the mean in order to improve recognition of the circalunar rhythm as explained in Section 4.1 .2 .

It is also recognized that the seasonal variation seen in the amplitude spectrum of the illuminance model is stronger than in the Skycalc model. This is caused because we only consider midnight values for the illuminance model, while for the Skycalc model nightly averages are calculated. Hence, the contribution of the peak NSB at midnight is smoothed out in time due to averaging, while it is fully captured for midnight data. The averaging effect is even stronger in winter when the Moon's contribution to the zenithal $<\mathrm{NSB}>$ is highest, because then the nights are longer. The seasonal signal is thus equalized throughout the year.

\subsection{Identifying the synodical month in the $<\mathrm{NSB}>$ data and quantifying its amplitude}

From our SQM data, we first calculate the nightly averages, i.e. $<$ NSB $>$, as described in section 2 and then perform an FFT analysis using the considerations from section 4.1. That way, we can clearly detect the circalunar rhythm at all our sites (see Table 4), be it rural or urban. However, the amplitude decreases from 1.55 to $0.33 \mathrm{mag}_{\mathrm{SQM}} \operatorname{arcsec}^{-2}$, corresponding to a factor of three on a linear scale, from the darkest to the brightest sites. A comparison between amplitude spectra of a typical urban and rural site is shown in Figure 7.

It is also recognized that at rural sites, harmonics of the main variation at a frequency $0.0339 \mathrm{~d}^{-1}$ 


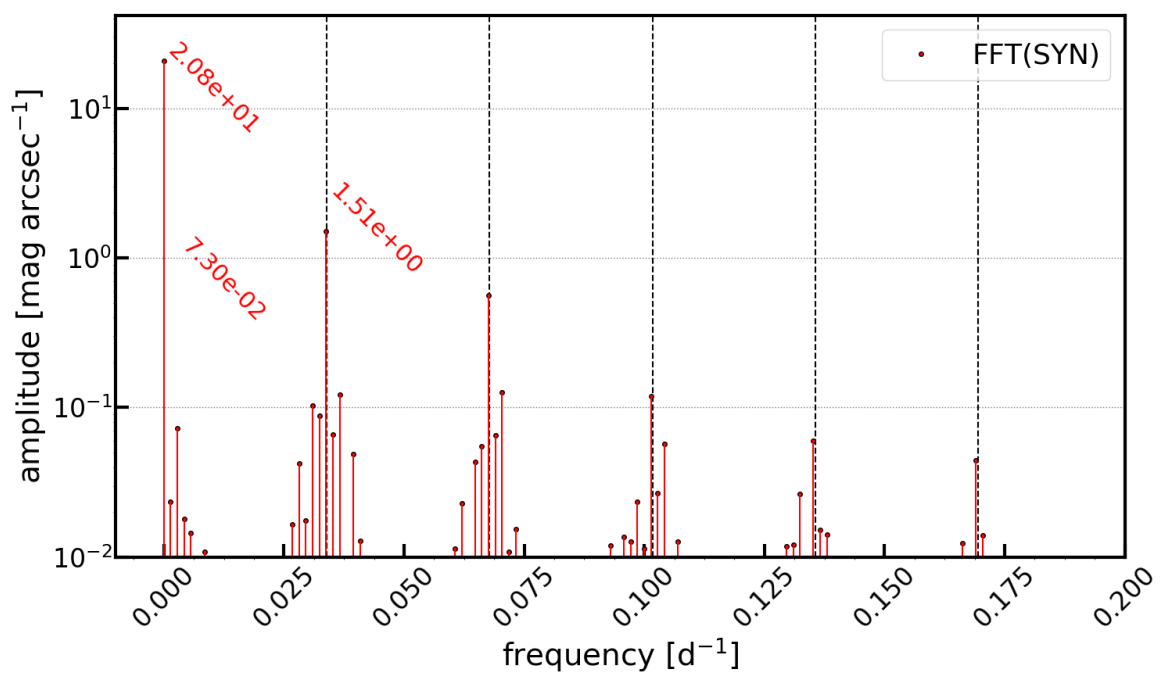

Figure 6. FFT amplitude spectrum of the Skycalc sky model as described in Section 3.2. The shown frequency range is limited to values between 0 and 0.2 . Labeled amplitudes (from left to right correspond to the mean $<\mathrm{NSB}>$ of the input data, the seasonal variation and the circalunar cycle.

can be identified up to 3rd order. In contrast, at urban sites, they perish in frequency noise, which increases with light pollution. In fact, the FFT noise roughly shows a bimodal distribution between rural and urban sites, as seen in Figure 8. This is, because day-to-day variations are more pronounced at urban, light-polluted sites due to backscattering of ALAN at clouds (Kyba et al., 2011, 2012, Puschnig et al., 2014a b; Aubé et al., 2016). Thus, the observed night sky brightness typically jumps between two preferred values; see e.g. (Posch et al., 2018, Figures A1a, A1b and 7). Since the noise as calculated here is dominated by high frequencies, i.e. roughly 64 percent of the frequencies correspond to periodic times of equal or less than five days, the same bimodality is seen here.

Another interesting feature seen in Figure 8 is that the scatter of the noise level increases along the $\langle\mathrm{NSB}>$ axis, i.e. darker sites show larger variance in the noise. The cause of this trend might be explained by findings of Kocifaj and Solano Lamphar (2014), who showed that the amplification factor due to clouds decreases with increasing city size and thus the level of light pollution.

\subsection{Relation between the circalunar amplitude and zenithal $<\mathrm{NSB}>$ for mid latitudes}

Previous observations have already qualitatively shown, that the circalunar rhythm steadily fades away with an increasing level of anthropogenic light at night. Using our FFT analysis, we can now quantify how ALAN affects the lunar cycle's degree of recognition. We do so by plotting the mean nightsky brightness, given by the amplitude at zero-frequency, against the amplitude of the circalunar rhythm. The result is shown in the left panel of Figure 9. A linear fit (see Equation 3) is found to be appropriate, with a scatter of only $0.062 \mathrm{magSQM} \operatorname{arcsec}^{-2}$. Extrapolation leads us to an $<\mathrm{NSB}>$ level of $16.5 \mathrm{mag}_{\mathrm{SQM}} \mathrm{arcsec}^{-2}$, the level at which the circalunar rhythm practically vanishes (at zenith) and is indistinguishable from the polluted nightsky.

$$
\mathrm{A}=0.322<\mathrm{NSB}>-5.324
$$

We may also convert the amplitudes in linear units, e.g. to luminance (see Equation 1), and then express the amplitude of the circalunar cycle as multiple of $\langle\mathrm{NSB}\rangle$, which we denote as circalunar contrast (CLC). As shown in the right panel of Figure 9, the contrast between the average $<$ NSB $>$ level and the darkening/brightening due to new/full moon ranges between 30 and 300 percent for our urban and rural sites respectively. 
Table 4. Summary of recognized features in the FFT amplitude spectrum for 23 SQM stations and the synthetic sky model (SYN). Column 1 is the station code, column 2 and 3, the average night sky brightness from the peak at zero frequency in units of $10^{-5} \mathrm{~W} \mathrm{~m}^{-2} \mathrm{sr}^{-1} \mu \mathrm{m}^{-1}$ and magSQM arcsec ${ }^{-2}$ respectively. The amplitude of the circalunar cycle in magSQM $\operatorname{arcsec}^{-2}$ is shown in column 4 and the circalunar contrast CLC, i.e. the amplitude expressed in percent of $\langle$ NSB $\rangle$, is found in column 5. The amplitude of the seasonal variation (bright winters, dark summers in units of magSQM $\operatorname{arcsec}^{-2}$ is given in column column 6 and the noise in $\mathrm{mag}_{\mathrm{SQM}} \operatorname{arcsec}^{-2}$ in the last column.

\begin{tabular}{ccccccc} 
COD & $<$ NSB $\rangle_{\mathrm{W}}$ & $\begin{array}{c}<\mathrm{NSB}\rangle_{\text {mag }} \\
(2)\end{array}$ & $\begin{array}{c}\mathrm{A} \\
(3)\end{array}$ & $\begin{array}{c}\text { CLC } \\
(4)\end{array}$ & $\begin{array}{c}\mathrm{S} \\
(6)\end{array}$ & $\begin{array}{c}\mathrm{N} \\
(7)\end{array}$ \\
\hline \hline LGO & 18.25 & 17.31 & 0.34 & 36.8 & 0.73 & 0.071 \\
LSM & 15.32 & 17.51 & 0.33 & 34.3 & 0.77 & 0.070 \\
WEL & 12.74 & 17.70 & 0.36 & 39.3 & 0.86 & 0.067 \\
LSW & 12.39 & 17.73 & 0.41 & 45.9 & 0.58 & 0.069 \\
STW & 10.31 & 17.93 & 0.45 & 50.0 & 0.49 & 0.069 \\
TRA & 8.90 & 18.09 & 0.46 & 52.8 & 0.55 & 0.061 \\
STY & 7.54 & 18.27 & 0.50 & 58.5 & 0.69 & 0.068 \\
PAS & 6.33 & 18.46 & 0.53 & 62.9 & 0.57 & 0.054 \\
BRA & 5.88 & 18.54 & 0.66 & 83.7 & 0.67 & 0.057 \\
GRI & 5.56 & 18.60 & 0.57 & 69.0 & 0.92 & 0.057 \\
VOE & 5.56 & 18.62 & 0.64 & 82.0 & 0.74 & 0.060 \\
FRE & 5.56 & 18.60 & 0.71 & 92.3 & 0.76 & 0.070 \\
MAT & 3.67 & 19.05 & 0.83 & 114.8 & 0.65 & 0.062 \\
MUN & 1.91 & 19.76 & 1.10 & 175.4 & 0.56 & 0.047 \\
GIS & 1.44 & 20.07 & 1.02 & 155.9 & 0.06 & 0.050 \\
ULI & 1.38 & 20.13 & 1.16 & 191.1 & 0.50 & 0.057 \\
KID & 1.12 & 20.37 & 1.19 & 204.8 & 0.09 & 0.040 \\
GRU & 1.07 & 20.39 & 1.34 & 243.6 & 0.38 & 0.036 \\
FEU & 0.84 & 20.66 & 1.22 & 207.6 & 0.07 & 0.057 \\
LOS & 0.83 & 20.86 & 1.30 & 243.6 & 0.01 & 0.059 \\
KRI & 0.73 & 20.81 & 1.16 & 191.1 & 0.07 & 0.049 \\
ZOE & 0.70 & 21.01 & 1.55 & 324.6 & 0.10 & 0.056 \\
BOD & 0.62 & 21.04 & 1.51 & 301.8 & 0.10 & 0.064 \\
SYN & 0.73 & 20.81 & 1.51 & 301.8 & 0.08 & 0.004
\end{tabular}




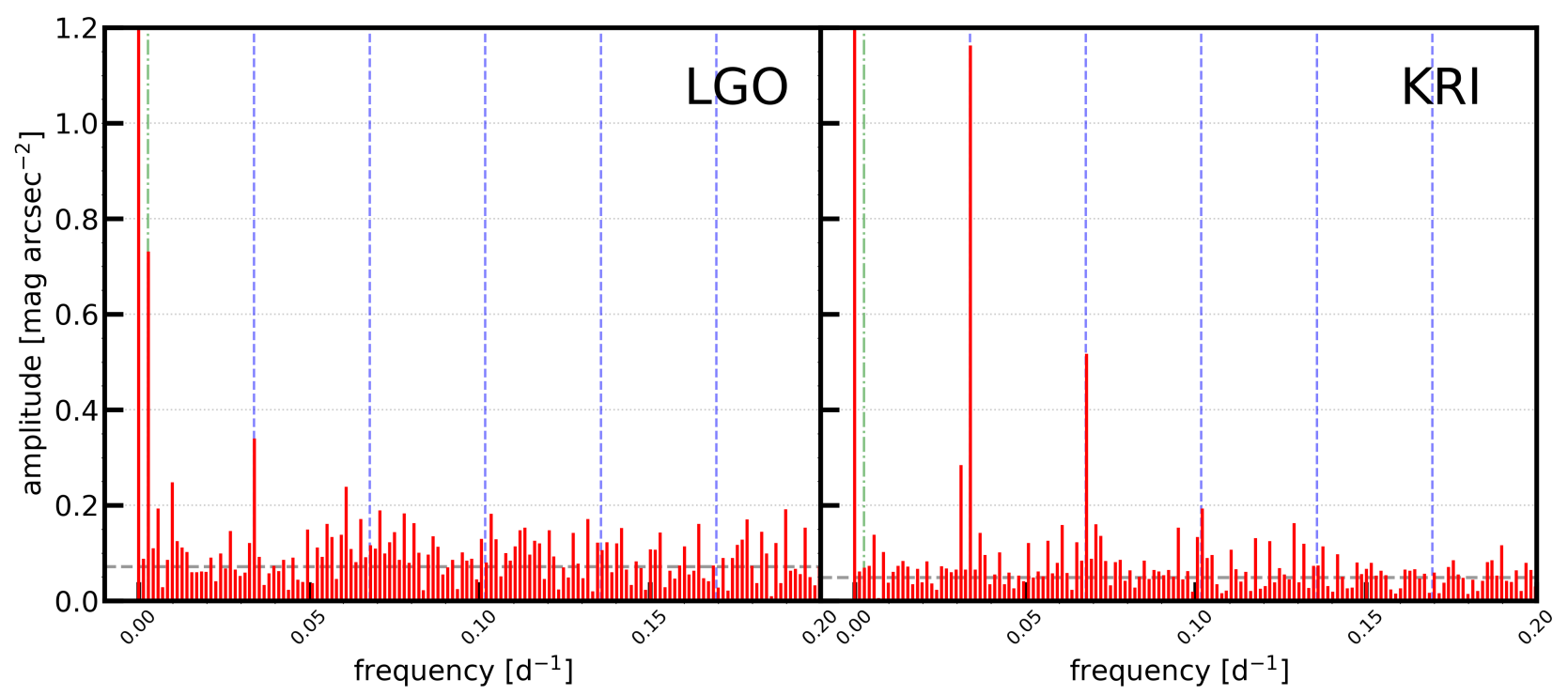

Figure 7. FFT amplitude spectrum of an urban (LGO) and rural (KRI) site. The shown frequency range is limited to values between 0 and 0.2 and the amplitude range is cut at 1.2 magsQM $\operatorname{arcsec}^{-2}$ in order to focus on amplitudes in the given frequency range, but excluding the peak at zero, i.e the mean nightsky brightness.

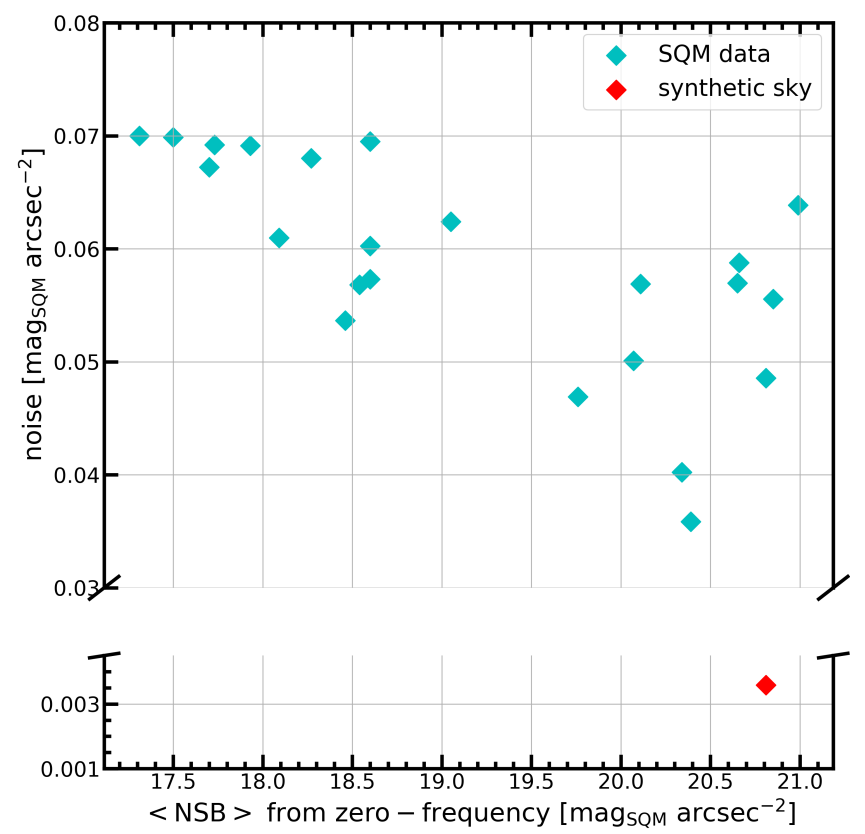

Figure 8. FFT noise increases with the level of light pollution. 

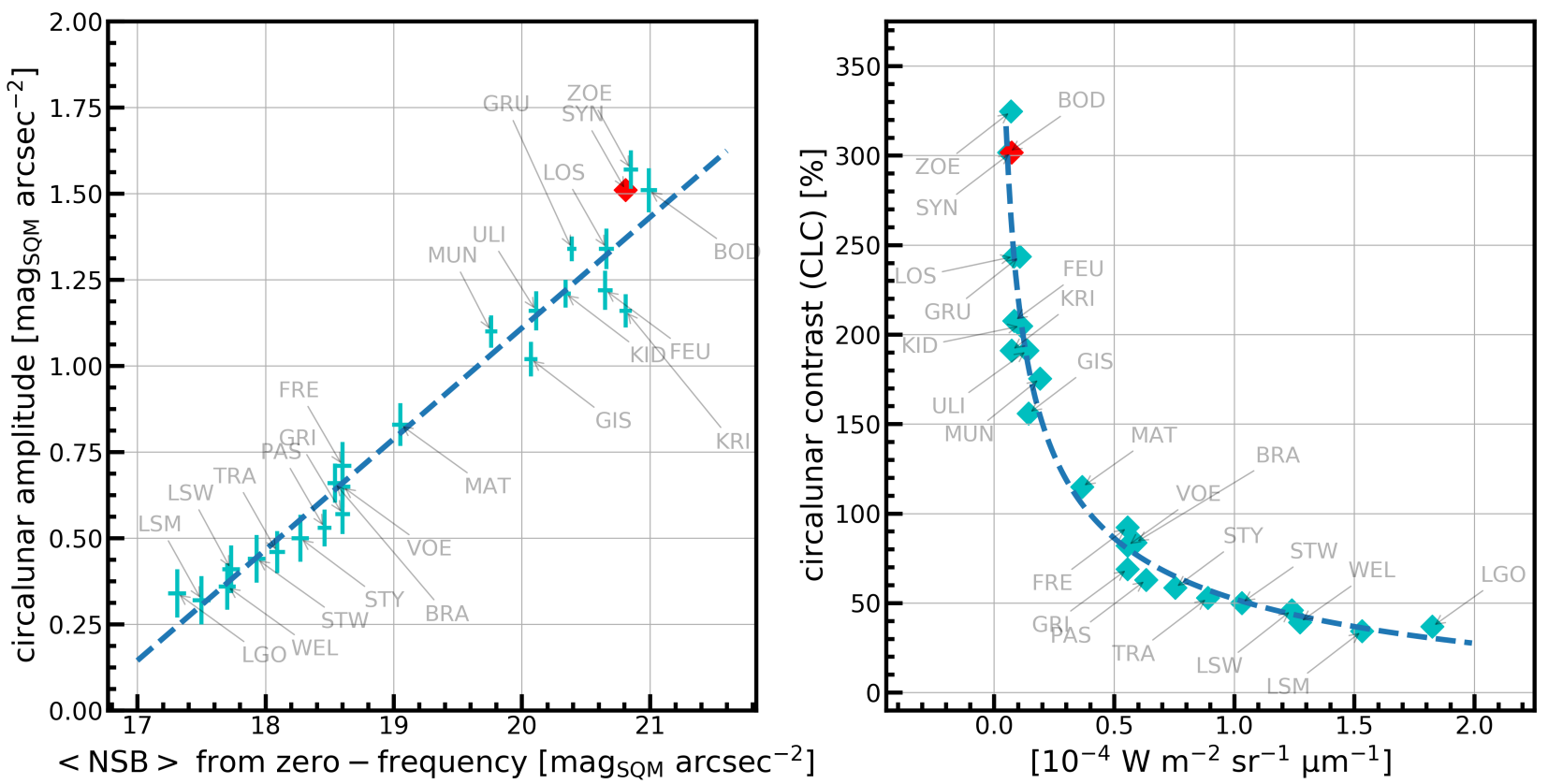

Figure 9. Left panel: Relation between the circalunar amplitude and zenithal $\langle\mathrm{NSB}\rangle$ for mid latitudes $\left(\sim 48^{\circ}\right)$. Individual points denote amplitudes derived through FFT analysis, with $\pm 1 \sigma$ errors found from the noise in the FFT amplitude spectra. The data is in good agreement with a linear relation with a scatter of only $0.06 \mathrm{magSQM}_{\mathrm{arcsec}}{ }^{-2}$. The red point denotes amplitude and $<\mathrm{NSB}>$ for our synthetic Skycalc sky model. Right panel: The same relation as shown in the left panel, but converted to luminance using Equation 1 and normalized to the moonless, zenithal luminance of the synthetic sky model, i.e. the contrast of the Moon's luminance at zenith against skyglow.

\subsection{Searching for other than lunar frequencies in the $<$ NSB $>$ data}

As seen in Sections 3 and 5.1 a naturally occuring seasonal variation caused by the changing height of the ecliptic (and thus the Moon) is expected. In our measurements, however, for the darkest, rural stations (FEU, KRI, LOS, ZOE, BOD), we do not recover any such seasonal variation at a significant level (compare Table 6). This is mainly because our $\langle\mathrm{NSB}>$ data includes overcast skies that weaken the amplitude of the signal in an unforeseeable way, and given the fact that we cover only two years, the signal easily vanishes.

On the other side, we do detect a very strong seasonal variation at all urban and also at most intermediate stations (compare Table 6). However, the amplitude of that signal is too large to be caused by the Moon. This oscillation might be the result of climatological effects that enhance ALAN during winters, i.e. combined effects of increased surface albedo and lower vegetation state (Wallner and Kocifaj, 2019). The observed seasonal variation might also be related to the aerosol optical depth (AOD). As demonstrated by Aubé (2015), the zenith radiance can increase several tens of times when optical depth is significantly lowered. However, disentangling the contribution of the several effects (surface albedo, vegetation, AOD) is beyond the scope of this paper and would require ancillary data products.

At urban sites (LGO, LSM, WEL, LSW, STY, PAS, BRA, GRI, VOE, MAT), we further detect a significant ( $>3$ sigma) signal with a periodic time of $\sim 100$ days. The cause of this roughly quaterly variation is unclear and was not reported previously. In our Table 6 we denote it as unknown.

We also search for weekly variations that might be associated with an increased human nighttime activity on weekends. Although we do see at our urban stations amplitudes on the order of $0.15-0.20$

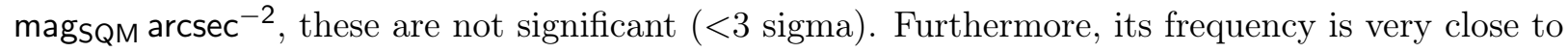
that of the third lunar harmonic. 


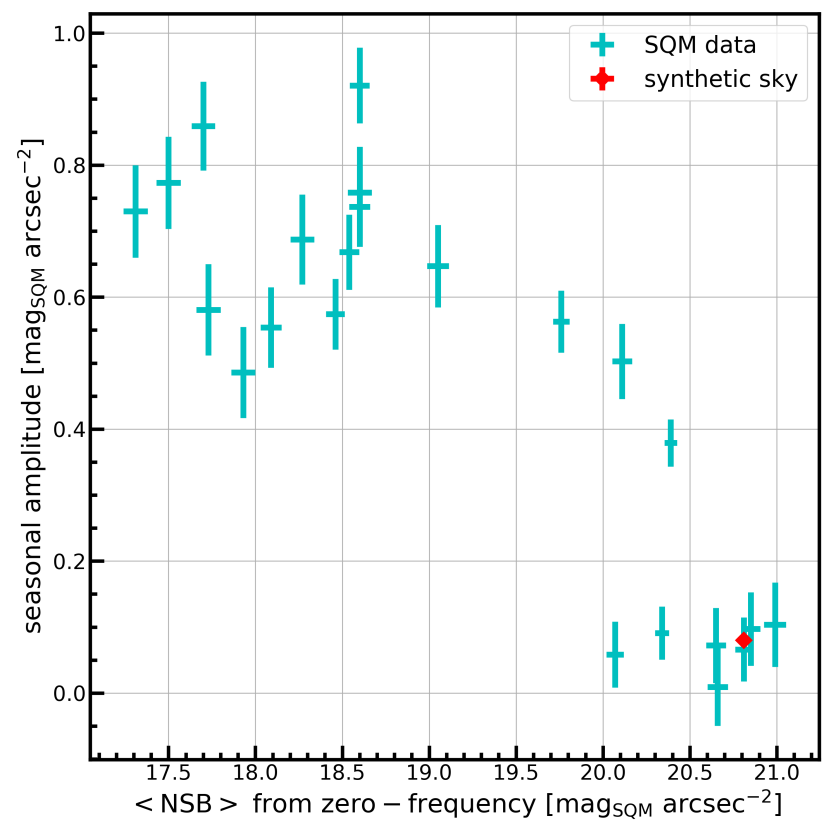

Figure 10. The amplitude of the seasonal oscillation against $\langle$ NSB $>$, both derived through FFT analysis of nightly $<\mathrm{NSB}>$ values. Each point corresponds to one of our SQM stations, with error bars indicating the $\pm 1 \sigma$ errors found from the noise in the FFT amplitude spectrum. The red point shows the location of our synthetic Skycalc sky model, indicating that for our SQM network the amplitude of the naturally occuring seasonal variation due to the Moon (its seasonal change of altitude) has an amplitude of less than $0.1 \mathrm{magSQM}_{\mathrm{S}} \operatorname{arsec}^{-2}$, which is too low to be detectable within the frequency noise as we see in our FFT amplitude spectra. Thus, no clear trend or relation is identified. The strong seasonal amplitudes seen at urban sites are thus caused by other seasonal effects such as changing albedo or vegetation state between winter and summer (Puschnig et al. in prep.). 


\section{Discussion and Conclusion}

Prior to a discussion of possible limitations of our method and implications of our findings in a wider context, we first compare our results to those of Bará (2016), who performed an SQM based FFT analysis of 14 Galician stations using a 1-year dataset. Bará (2016) have previously defined the moonlight factor (y) as the ratio of power densities between the fundamental circalunar frequency and the zero frequency. Note that they used double-sided power density spectra, while we use single-sided amplitude spectra. The power spectra of Bará (2016) thus show negative and positive frequency components $k>0$ with heights of $A_{k}^{2} / 4$ compared to our amplitudes (A) in Table 4 and at a height of $A_{0}^{2}$ for the zero-frequency component $\left(<\mathrm{NSB}>_{\text {mag }}\right.$ ). They reported ranges for the moonlight factor between $0.2-0.3 \times 10^{-3}$ for urban sites, $1.5-2.4 \times 10^{-3}$ for dark rural sites and values in-between for intermediate regions. A compilation of the moonlight factor calculated for our sites is shown in Table 5. We find that the moonlight factor derived through our FFT methodology and data is almost a factor of 2 lower than those reported in Bará (2016). Although it is expected that the Moon's impact on zenithal NSBs in Galicia is stronger than in Upper Austria, because the Galician network's geographic latitude is lower by $\sim 5^{\circ}$ and thus the ecliptic and Moon closer to zenith, a factor of 2 seems to be relatively high. We argue that the relatively large difference is mainly caused because Bará (2016) take into account NSB measurements obtained at midnight only rather than averaging over dark time hours as we do. As a result - in analogy to the explanation in Section 5.1 for nightly averages peak values that occur at midnight are smoothed in time, which is also the reason why we do not capture the seasonal variation (compare Figure 10). On the other hand, averaging over dark times has the advantage of providing a better definition and thus less scatter of the amplitudes over several months, because a single nightly measurement may easily be affected by short-term perturbations such as cloud cover, which may impact NSB measurements in a complex way: Considering that backscattering of moonlight on scattered clouds (in zenith) exists in analogy to previous findings of Kyba et al. (2011, 2012); Puschnig et al. (2014a b), clouds may enhance the zenithal NSB on the one hand, but it may also lower the zenithal NSB on the other hand, e.g for fully overcast skies, similar to previous findings of (Posch et al., 2018; Jechow et al., 2019).

However, recognition of the circalunar amplitude as performed by Bará (2016) is affected by spectral leakage that lowers the amplitude (compared to our approach) due to smearing out of the signal over multiple frequencies, as explained in Section 4.1.4. Additionally, Bará (2016) use only 1-year of input data, leading to lower frequency resolution and thus even more leakage, as explained in Section 4.1.3.

We conclude that a combination of these effects causes the factor 2 discrepancy, but we stress that the linear relation in Figure 9 , its intercept mainly, would not change by a factor 2 for geographic locations similar to those of the Galician network. This is shown in the following.

In principle, it is expected that the relation in Figure 9 shifts towards lower circalunar amplitudes for geographic latitudes north of $48^{\circ}$ and towards higher amplitudes otherwise. In order to asses how much the relation's intercept may shift, we perform FFT analysis of 2-year Skycalc models calculated for different latitudes. For the first test, we choose a latitude of $\mathrm{N} 36^{\circ}$ (e.g. Gibraltar). The derived amplitude of the circalunar cycle is $1.61 \mathrm{mag}_{\mathrm{SQM}} \operatorname{arcsec}^{-2}$, which is only $\sim 0.1 \mathrm{mag}_{\mathrm{SQM}} \operatorname{arcsec}^{-2}$ higher than for the synthetic models calculated for our network. For the second test, we choose a latitude of $\mathrm{N} 60^{\circ}$ (e.g. slightly north of Stockholm). However, we caution that for latitudes above $51.5^{\circ}$, during summer there are no dark times with the sun being more than $15^{\circ}$ below the horizon. As a result, gaps in the input data limit the recoverability of the circalunar amplitude, as explained in Section 4.1.2. In that case we thus find an amplitude of $1.02 \mathrm{magSQM}_{\mathrm{arcsec}}{ }^{-2}$ only, which is mainly due to the large summer gaps accounting for roughly 25 percent of the input data. From these tests we conclude that our relation between the circalunar amplitude and $<\mathrm{NSB}>$ is at least valid for latitudes between $\sim 40-50^{\circ}$.

Our linear relation between zenithal $<\mathrm{NSB}>$ and the circalunar amplitude in Figure 9 implies 
that the circa-monthly variation of moonlight is still traceable over large areas, not only in Upper

Austria, but also in many other countries (compare light pollution atlas by Falchi et al. (2016)). The circalunar rhythm is thus expected to practically vanish due to ALAN only in the innermost parts of major cities where the zenithal NSB may exceed 16.5 magSQM $\operatorname{arcsec}^{-2}$. However, at the same time, it is recognized that the contribution of the Moon to the total zenithal NSB (i.e. contrast) is largely reduced due to ALAN, namely by a factor of $\sim 1 / 9$ for urban areas (e.g. Linz with $\sim 200,000$ inhabitants), a factor of $\sim 1 / 3$ for small towns with less than 10,000 inhabitants (e.g. Freistadt or Mattighofen) and still up to $\sim 1 / 2$ for one of our rural stations (e.g. Münzkirchen, a village with less than 2,600 inhabitants). Only two of our sites, both situated in national parks (Bodinggraben and Zöblboden), show natural circalunar amplitudes.

Finally, we discuss implications of the SQM's spectral bandpass that covers a range of $\sim 300-680 \mu \mathrm{m}$. Although this is very close to the so called photosynthetically active radiation, i.e. the spectral range between $400-700 \mu \mathrm{m}$ to which photosynthetic organisms are sensitive, we caution that within that spectral range, Chlorophyll - the most abundant plant pigment - has a sensitivity curve that is very different from the SQM's sensitivity curve shown in Figure 1 . Rather than being mostly sensitive to green photons, Chlorophyll is mostly sensitive to red and blue photons. The exact degradation of the circalunar rhythm as recognized by photosynthetic organisms may thus be different from what we observe using SQMs. However, other species such as e.g. ocean fish, that have

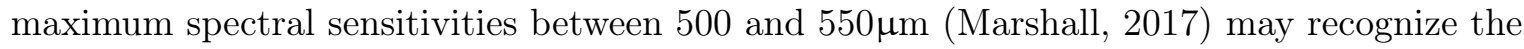
degradation of the circalunar cycle exactly as described in our paper. How they are affected by a decreased amplitude of the circa-monthly signal is yet to be shown in future (chrono)biological studies.

\section{Summary}

We studied the circalunar periodicity via FFT analysis of night-time averages of zenithal NSB measurements obtained during the years 2016 and 2017 through a network of 23 SQMs located in Upper Austria at a latitude of $\sim 48^{\circ}$. Models of the sky (Noll et al., 2012, Jones et al., 2013) and the Moon (Seidelmann, 1992) were used as a reference of ideal conditions and to develop an optimal methodology for the recognition of the circalunar periodicity. The following conclusions are drawn from our study:

- A tight linear relation between $<\mathrm{NSB}>$ given in $\operatorname{magSQM}_{\mathrm{S}} \operatorname{arsec}^{-2}$ and the circalunar amplitude is found (see Figure 9). This relation indicates that for sites with $<\mathrm{NSB}>$ brighter than 16.5

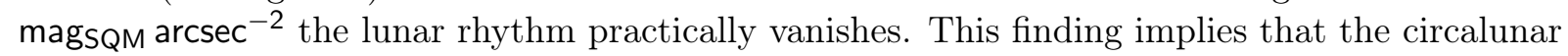
rhythm is still detectable (within the broad bandpass of the SQM) at most places around the globe.

- However, the circalunar contrast in zenith is largely reduced compared to ALAN-free sites. In the state capital of Linz ( 200,000 inhabitants) the Moon's contribution to zenithal $<\mathrm{NSB}>$ is reduced by a factor of $1 / 9$. For small towns (e.g. Freistadt or Mattighofen) with less than 10,000 inhabitants, we find that the circalunar contrast in zenith is lowered by a factor of $1 / 3$ due to ALAN and even at one of our rural sites, Münzkirchen, a village with less than 2,600 inhabitants, the circalunar zenithal contrast is reduced to a level of $1 / 2$ compared to ALAN-free conditions.

- Only two of our sites, both situated in national parks (Bodinggraben and Zöblboden), show natural circalunar amplitudes.

- At our urban sites we further detect a strong seasonal signal that is linked to the amplification of anthropogenic skyglow during the winter months due to combined effects of enhanced albedo (due to snow) and a lower vegetation state (Puschnig et al. in prep). 
Table 5. Station codes and zero-frequency $<\mathrm{NSB}>$ in units of magSQM $\operatorname{arcsec}^{-2}$ are shown in columns 1 and 2. Calculations of the moonlight factor (y) as defined in Bará (2016) are given in column 3

\begin{tabular}{ccc} 
COD & $\begin{array}{c}\text { NSB }> \\
(1)\end{array}$ & $\begin{array}{c}y \times 10^{3} \\
(2)\end{array}$ \\
\hline \hline & urban & \\
LGO & 17.31 & 0.10 \\
LSM & 17.51 & 0.09 \\
WEL & 17.7 & 0.10 \\
LSW & 17.73 & 0.13 \\
STW & 17.93 & 0.16 \\
TRA & 18.09 & 0.16 \\
STY & 18.27 & 0.19 \\
& intermediate & \\
PAS & 18.46 & 0.21 \\
BRA & 18.54 & 0.32 \\
GRI & 18.6 & 0.23 \\
VOE & 18.62 & 0.30 \\
FRE & 18.6 & 0.36 \\
MAT & 19.05 & 0.47 \\
& rural & \\
MUN & 19.76 & 0.77 \\
GIS & 20.07 & 0.65 \\
ULI & 20.13 & 0.83 \\
KID & 20.37 & 0.85 \\
GRU & 20.39 & 1.08 \\
FEU & 20.66 & 0.87 \\
LOS & 20.86 & 0.97 \\
KRI & 20.81 & 0.78 \\
ZOE & 21.01 & 1.36 \\
BOD & 21.04 & 1.29 \\
SYN & 20.81 & 1.32 \\
& & \\
& &
\end{tabular}


- At urban sites we further detect a significant ( $>3$ sigma) signal with a periodic time of $\sim 100$ days. The cause of this roughly quaterly variation is unclear and was not reported previously.

\section{Acknowledgements}

We dedicate this work to Thomas Posch, our most valued colleague, mentor and dearest friend, who passed away during the development phase of this manuscript. This paper would have never been written without Thomas, as it was him who first introduced Johannes and Stefan to this stunning field of research many years ago. He will be missed by us, not only because of his expertise and keen mind, but also because of his cordiality.

We acknowledge support by the provincial government of Upper Austria, in particular we thank Heribert Kaineder and Martin Waslmaier for their dedication and tireless efforts to establish a network of SQMs in Upper Austria, and share the data with the public.

We are grateful to the referee for his/her constructive input.

This research further made use of SciPy (Jones et al., 2001) and NumPy (Van Der Walt et al. 2011), two Python packages that make life as a scientist easier.

\section{References}

Harold Munro Fox and John Stanley Gardiner. Lunar periodicity in reproduction. Proceedings of the Royal Society of London. Series B, Containing Papers of a Biological Character, 95(671):523-550, 1924. doi: 10.1098/rspb.1924.0004. URL https://royalsocietypublishing.org/doi/abs/10.1098/rspb.1924.0004.

Florian Raible, Hiroki Takekata, and Kristin Tessmar-Raible. An overview of monthly rhythms and clocks. Frontiers in Neurology, 8:189, 2017. ISSN 1664-2295. doi: 10.3389/fneur.2017.00189. URL https://www.frontiersin.org/article/10.3389/fneur.2017.00189.

Jennifer A. Clarke. Moonlight's influence on predator/prey interactions between short-eared owls (asio flammeus) and deermice (peromyscus maniculatus). Behavioral Ecology and Sociobiology, 13 (3):205-209, Sep 1983.

Tamaki Shimose, Kotaro Yokawa, and Katsunori Tachihara. Higher catch rates around the full moon for blue marlin, makaira nigricans, in a diurnal trolling fishery. Bulletin of Marine Science, 89(3): 759-765, 2013.

Chris Fallows, Monique Fallows, and Neil Hammerschlag. Effects of lunar phase on predator-prey interactions between white shark (carcharodon carcharias) and cape fur seals (arctocephalus pusillus pusillus). Environmental Biology of Fishes, 99(11):805-812, Nov 2016. ISSN 1573-5133. doi: 10.1007/s10641-016-0515-8. URL https://doi.org/10.1007/s10641-016-0515-8.

H. H. Kolb. The effect of moonlight on activity in the wild rabbit (oryctolagus cuniculus). Journal of Zoology, 228(4):661-665, 1992.

Noga Kronfeld-Schor, Davide Daominoni, Horacio De la Iglesia, Oren Levy, Erik D. Herzog, Tamar Dayan, and Charlotte Helfrich-Forster. Chronobiology by moonlight. Proceedings of the Royal Society B: Biological Sciences, 280(20123088), 2013. doi: 10.1098/rspb.2012.3088.

Akihiro Takemura, Satomi Ueda, Nanae Hiyakawa, and Yoshiaki Nikaido. A direct influence of moonlight intensity on changes in melatonin production by cultured pineal glands of the golden rabbitfish, siganus guttatus. Journal of Pineal Research, 40(3):236-241, 2006. doi:

10.1111/j.1600-079X.2005.00306.x. URL https://onlinelibrary.wiley.com/doi/abs/10.1111/j.1600-079X.2005.00306.x. 
F. Papi and L. Pardi. On the lunar orientation of sandhoppers (amphipoda talitridae). The Biological Bulletin, 124(1):97-105, 1963. doi: 10.2307/1539571. URL https://doi.org/10.2307/1539571.

Karl von Frisch. The Dance Language and Orientation of Bees. Harvard University Press, 1993.

M. Dacke, M. J. Byrne, E. Baird, C. H. Scholtz, and E. J. Warrant. How dim is dim? precision of the celestial compass in moonlight and sunlight. Philosophical Transactions of the Royal Society B: Biological Sciences, 366(1565):697-702, 2011. doi: 10.1098/rstb.2010.0191.

Erwin Bünning and Ilse Moser. Interference of moonlight with the photoperiodic measurement of time by plants, and their adaptive reaction. Proceedings of the National Academy of Sciences, 62: 1018-1022, 1969. doi: 10.1073/pnas.62.4.1018.

Mossadok Ben-Attia, Alain Reinberg, Michael H. Smolensky, Wafa Gadacha, Achraf Khedaier, Mamane Sani, Yvan Touitou, and Néziha Ghanem Boughamni. Blooming rhythms of cactus cereus peruvianus with nocturnal peak at full moon during seasons of prolonged daytime photoperiod. Chronobiology International, 33(4):419-430, 2016. doi: 10.3109/07420528.2016.1157082. URL https://doi.org/10.3109/07420528.2016.1157082. PMID: 27030087.

Juliane Zantke, Tomoko Ishikawa-Fujiwara, Enrique Arboleda, Claudia Lohs, Katharina Schipany, Natalia Hallay, Andrew?D Straw, Takeshi Todo, and Kristin Tessmar-Raible. Circadian and circalunar clock interactions in a marine annelid. Cell Reports, 5(1):99-113, Oct 2013. ISSN 2211-1247. doi: 10.1016/j.celrep.2013.08.031. URL https://doi.org/10.1016/j.celrep.2013.08.031.

Kim S. Last, Laura Hobbs, Jorgen Berge, Andrew S. Brierley, and Cottier Finlo. Moonlight drives ocean-scale mass vertical migration of zooplankton during the arctic winter. Current Biology, 26: 244-251, 2016. doi: 10.1016/j.cub.2015.11.038.

M. Zimecki. The lunar cycle: Effects on human and animal behavior and physiology. Postepy Hig Med Dosw. (Online), 60:1-7, 022006.

Alain Reinberg, Michael H. Smolensky, and Yvan Touitou. The full moon as a synchronizer of circa-monthly biological rhythms: Chronobiologic perspectives based on multidisciplinary naturalistic research. Chronobiology International, 33(5):465-479, 2016.

Sung Ping Law. The regulation of menstrual cycle and its relationship to the moon. Acta Obstetricia et Gynecologica Scandinavica, 65(1):45-48, 1986. doi: 10.3109/00016348609158228.

Christian Cajochen, Songül Altanay-Ekici, Mirjam Münch, Silvia Frey, Vera Knoblauch, and Anna Wirz-Justice. Evidence that the lunar cycle influences human sleep. Current Biology, 23(15): 1485-1488, 2013.

Maren Cordi, Sandra Ackermann, Frederik W. Bes, Francina Hartmann, Boris N. Konrad, Lisa Genzel, Marcel Pawlowski, Axel Steiger, Hartmut Schulz, Björn Rasch, and Martin Dresler. Lunar cycle effects on sleep and the file drawer problem. Current Biology, 24(12):R549-R550, 032014. doi: $10.1016 /$ j.cub.2014.05.017.

Christian Cajochen, Songül Altanay-Ekici, Mirjam Münch, Silvia Frey, Vera Knoblauch, and Anna Wirz-Justice. Reply to cordi et al. Current biology, 24:R795, 092014.

Christopher C. M. Kyba, Kai Pong Tong, Jonathan Bennie, Ignacio Birriel, Jennifer J. Birriel, Andrew Cool, Arne Danielsen, Thomas W. Davies, Peter N. den Outer, William Edwards, Rainer Ehlert, Fabio Falchi, Jürgen Fischer, Andrea Giacomelli, Francesco Giubbilini, Marty Haaima, Claudia Hesse, Georg Heygster, Franz Hölker, Richard Inger, Linsey J. Jensen, Helga U. Kuechly, 
John Kuehn, Phil Langill, Dorien E. Lolkema, Matthew Nagy, Miguel Nievas, Nobuaki Ochi, Emil Popow, Thomas Posch, Johannes Puschnig, Thomas Ruhtz, Wim Schmidt, Robert Schwarz, Axel Schwope, Henk Spoelstra, Anthony Tekatch, Mark Trueblood, Constance E. Walker, Michael Weber, Douglas L. Welch, Jaime Zamorano, and Kevin J. Gaston. Worldwide variations in artificial skyglow. Scientific Reports, 5, 022015.

Franz Hölker, Christian Wolter, Elizabeth K. Perkin, and Klement Tockner. Light pollution as a biodiversity threat. Trends in Ecology $\& 3$ Evolution, 25(12):681-682, Dec 2010. ISSN 0169-5347. doi: 10.1016/j.tree.2010.09.007. URL https://doi.org/10.1016/j.tree.2010.09.007.

Kevin J. Gaston, Marcel E. Visser, and Franz Hölker. The biological impacts of artificial light at night: the research challenge. Philosophical Transactions of the Royal Society B: Biological Sciences, 370(1667):20140133, 2015. doi: 10.1098/rstb.2014.0133. URL https://royalsocietypublishing.org/doi/abs/10.1098/rstb.2014.0133

Alessandro Manfrin, Gabriel Singer, Stefano Larsen, Nadine Weiß, Roy H. A. van Grunsven, Nina-Sophie Weiß, Stefanie Wohlfahrt, Michael T. Monaghan, and Franz Hölker. Artificial light at night affects organism flux across ecosystem boundaries and drives community structure in the recipient ecosystem. Frontiers in Environmental Science, 5:61, 2017. ISSN 2296-665X. doi: 10.3389/fenvs.2017.00061. URL https://www.frontiersin.org/article/10.3389/fenvs.2017.00061.

Sibylle Schroer and Franz Hölker. Impact of Lighting on Flora and Fauna, pages 1-33. Springer International Publishing, 01 2016. doi: 10.1007/978-3-319-00295-8_42-1.

Thomas W. Davies, Jonathan Bennie, Richard Inger, and Kevin J. Gaston. Artificial light alters natural regimes of night-time sky brightness. Scientific Reports, 3:1722 EP -, 04 2013. URL https://doi.org/10.1038/srep01722.

P. Cinzano. Night Sky Photometry with Sky Quality Meter. Technical report, Dipartimento di Astronomia, Vicolo dell Osservatorio 2, I-35100 Padova, Italy, Istituto di Scienza e Tecnologia dell Inquinamento Luminoso, Via Roma 13, I-36106 Thiene, Italy, 2005. URL http://www.inquinamentoluminoso.it/download/sqmreport.pdf. first draft, ISTIL Internal Report n. 9, v.1.4 2005.

Salvador Bará, Carlos E. Tapia, and Jaime Zamorano. Absolute radiometric calibration of tess-w and sqm night sky brightness sensors. Sensors, 19(6), 2019. ISSN 1424-8220. doi: 10.3390/s19061336. URL https ://www.mdpi.com/1424-8220/19/6/1336.

T. Posch, F. Binder, and J. Puschnig. Systematic measurements of the night sky brightness at 26 locations in Eastern Austria. Journal of Quantitative Spectroscopy and Radiative Transfer, 211: 144-165, May 2018. doi: 10.1016/j.jqsrt.2018.03.010.

Salvador Bará. Anthropogenic disruption of the night sky darkness in urban and rural areas. Royal Society Open Science, 3(10):160541, 2016. doi: 10.1098/rsos.160541.

Salvador Bará. Variations on a classical theme: On the formal relationship between magnitudes per square arcsecond and luminance. International Journal of Sustainable Lighting, 19, 10 2017. doi: 10.26607/ijsl.v19i2.77.

R. H. Garstang. Model for artificial night-sky illumination. Publications of the Astronomical Society of the Pacific, 98:364, mar 1986. doi: 10.1086/131768. URL https://doi.org/10.1086\%2F131768.

P. Kenneth Seidelmann. Explanatory Supplement to the Astronomical Almanac. University Science Books, 8 1992. ISBN 0935702687. 
Bruce Hapke, Robert Nelson, and William Smythe. The opposition effect of the moon: Coherent backscatterandshadow hiding. Icarus, 133(1):89 - 97, 1998. ISSN 0019-1035. doi:

https://doi.org/10.1006/icar.1998.5907. URL

http://www.sciencedirect.com/science/article/pii/S0019103598959073.

S. Noll, W. Kausch, M. Barden, A. M. Jones, C. Szyszka, S. Kimeswenger, and J. Vinther. An atmospheric radiation model for Cerro Paranal. I. The optical spectral range. Astronomy \& Astrophysics, 543:A92, July 2012. doi: 10.1051/0004-6361/201219040.

A. Jones, S. Noll, W. Kausch, C. Szyszka, and S. Kimeswenger. An advanced scattered moonlight model for Cerro Paranal. Astronomy \& Astrophysics, 560:A91, December 2013. doi:

10.1051/0004-6361/201322433.

Andrea Bertolo, Renata Binotto, Sergio Ortolani, and Simone Sapienza. Measurements of night sky brightness in the veneto region of italy: Sky quality meter network results and differential photometry by digital single lens reflex. Journal of Imaging, 5(5), 2019. ISSN 2313-433X. doi: 10.3390/jimaging5050056. URL https ://www.mdpi.com/2313-433X/5/5/56.

Stefan Van Der Walt, S Chris Colbert, and Gael Varoquaux. The numpy array: a structure for efficient numerical computation. Computing in Science \& Engineering, 13(2):22-30, 2011.

C. Munteanu, C. Negrea, M. Echim, and K. Mursula. Effect of data gaps: comparison of different spectral analysis methods. Annales Geophysicae, 34:437-449, April 2016. doi: 10.5194/angeo-34-437-2016.

Christopher C. M. Kyba, Thomas Ruhtz, JÃijrgen Fischer, and Franz HÃúlker. Cloud coverage acts as an amplifier for ecological light pollution in urban ecosystems. PLOS ONE, 6(3):1-9, 032011. doi: 10.1371/journal.pone.0017307. URL https://doi.org/10.1371/journal.pone.0017307.

C. C. M. Kyba, T. Ruhtz, J. Fischer, and F. HÃulker. Red is the new black: how the colour of urban skyglow varies with cloud cover. Monthly Notices of the Royal Astronomical Society, 425(1): 701-708, 2012. doi: 10.1111/j.1365-2966.2012.21559.x. URL https://onlinelibrary.wiley.com/doi/abs/10.1111/j.1365-2966.2012.21559.x.

J. Puschnig, T. Posch, and S. Uttenthaler. Night sky photometry and spectroscopy performed at the Vienna University Observatory. Journal of Quantitative Spectroscopy and Radiative Transfer, 139: 64-75, May 2014a. doi: 10.1016/j.jqsrt.2013.08.019.

J. Puschnig, A. Schwope, T. Posch, and R. Schwarz. The night sky brightness at Potsdam-Babelsberg including overcast and moonlit conditions. Journal of Quantitative Spectroscopy and Radiative Transfer, 139:76-81, May 2014b. doi: 10.1016/j.jqsrt.2013.12.011.

M. Aubé, M. Kocifaj, J. Zamorano, H.A. Solano Lamphar, and A. Sanchez de Miguel. The spectral amplification effect of clouds to the night sky radiance in madrid. Journal of Quantitative Spectroscopy and Radiative Transfer, 181:11 - 23, 2016. ISSN 0022-4073. doi: https://doi.org/10.1016/j.jqsrt.2016.01.032. URL http://www.sciencedirect.com/science/article/pii/S0022407315301849. Using remote sensing to better understand light pollution (Light Pollution Theory Modelling and Measurements 2015).

Miroslav Kocifaj and HÃl'ctor Antonio Solano Lamphar. Quantitative analysis of night skyglow amplification under cloudy conditions. Monthly Notices of the Royal Astronomical Society, 443(4): 3665-3674, 08 2014. ISSN 0035-8711. doi: 10.1093/mnras/stu1301. URL https://doi.org/10.1093/mnras/stu1301. 
Stefan Wallner and Miroslav Kocifaj. Impacts of surface albedo variations on the night sky brightness âĂ a numerical and experimental analysis. Journal of Quantitative Spectroscopy and Radiative Transfer, 239:106648, 2019. ISSN 0022-4073. doi: https://doi.org/10.1016/j.jqsrt.2019.106648. URL http://www . sciencedirect.com/science/article/pii/S0022407319304893.

Martin Aubé. Physical behaviour of anthropogenic light propagation into the nocturnal environment. Philosophical Transactions of the Royal Society B: Biological Sciences, 370(1667):20140117, 2015. doi: 10.1098/rstb.2014.0117. URL https://royalsocietypublishing.org/doi/abs/10.1098/rstb.2014.0117.

Andreas Jechow, Franz Hölker, and Christopher C. M. Kyba. Using all-sky differential photometry to investigate how nocturnal clouds darken the night sky in rural areas. Scientific Reports, 9(1):1391, 2019. ISSN 2045-2322. doi: 10.1038/s41598-018-37817-8. URL https://doi.org/10.1038/s41598-018-37817-8.

Fabio Falchi, Pierantonio Cinzano, Dan Duriscoe, Christopher C. M. Kyba, Christopher D. Elvidge, Kimberly Baugh, Boris A. Portnov, Nataliya A. Rybnikova, and Riccardo Furgoni. The new world atlas of artificial night sky brightness. Science Advances, 2(6), 2016. doi: 10.1126/sciadv.1600377. URL https://advances .sciencemag.org/content/2/6/e1600377

Justin Marshall. Vision and lack of vision in the ocean. Current Biology, 27(11):R494 - R502, 2017. ISSN 0960-9822. doi: https://doi.org/10.1016/j.cub.2017.03.012. URL http://www.sciencedirect.com/science/article/pii/S0960982217302816

E. Jones, T. Oliphant, P. Peterson, and Others. SciPy: Open source scientific tools for python, 2001. URL http://www . scipy.org/. 




Figure 11. The panels show cutouts of the FFT amplitude spectra ranging from 0 to $0.1 \mathrm{~d}^{-1}$ for 23 locations in Upper Austria, sorted by increasing $<\mathrm{NSB}>$ values, i.e. decreasing light pollution. The amplitude spectra were obtained from a two year long dataset (2016-2017) of nightly $<$ NSB $>$ values. For comparison, an amplitude spectrum of a cloud-free synthetic sky model as described in section 3.2 is shown in the bottom right panel (SYN). The peak at zero frequency, i.e. $\langle\mathrm{NSB}\rangle$, is indicated by a black dotted line, the seasonal variation $\frac{1}{365} \mathrm{~d}^{-1}$ is marked with a green dash-dotted line, the expected lunar synodic frequency of $\frac{1}{29.5} \mathrm{~d}^{-1}$ and its first harmonic are marked with blue, dashed lines and the mean noise level is shown as horizontal dashed line. 
Table 6. Summary of significant $\left(\frac{S}{N}>3\right)$ peaks in the FFT amplitude spectra. No windowing function was applied, instead we truncated the input time series at the edges in order to avoid discontinuities with respect to the lunar synodic month. That way, the circalunar amplitude could be recovered with highest accuracy. However, other amplitudes such as the one attributed to the seasonal variation are only lower limits in most cases. We found that the application of a Hanning window leads to an increase of the seasonal amplitude between 0.05 and $0.1 \mathrm{mag}_{\mathrm{SQM}} \operatorname{arcsec}^{-2}$.

\begin{tabular}{|c|c|c|c|c|c|c|c|c|c|c|c|}
\hline $\begin{array}{l}\text { COD } \\
\text { (1) }\end{array}$ & $\begin{array}{l}v \\
(2)\end{array}$ & $\begin{array}{l}\mathrm{T} \\
(3)\end{array}$ & $\begin{array}{l}\text { A } \\
(4)\end{array}$ & $\begin{array}{l}\mathrm{S} / \mathrm{N} \\
(5)\end{array}$ & $\begin{array}{l}\text { note } \\
(6)\end{array}$ & $\begin{array}{l}\text { COD } \\
\text { (1) }\end{array}$ & $\begin{array}{l}v \\
(2)\end{array}$ & $\begin{array}{l}\mathrm{T} \\
(3)\end{array}$ & $\begin{array}{l}\text { A } \\
(4)\end{array}$ & $\begin{array}{l}\mathrm{S} / \mathrm{N} \\
(5)\end{array}$ & $\begin{array}{l}\text { note } \\
(6)\end{array}$ \\
\hline$\overline{\mathrm{LGO}}$ & $0.00 \mathrm{e}+00$ & $\overline{\text { inf }}$ & 17.31 & 244.0 & mean & BRA & $0.00 \mathrm{e}+00$ & $\overline{\text { inf }}$ & 18.54 & 326.3 & mean \\
\hline LGO & $2.83 \mathrm{e}-03$ & 353.0 & 0.73 & 10.3 & seasonal & BRA & $2.82 \mathrm{e}-03$ & 354.0 & 0.67 & 11.8 & seasonal \\
\hline LGO & $9.92 \mathrm{e}-03$ & 100.9 & 0.24 & 3.4 & unknown & BRA & $5.65 \mathrm{e}-03$ & 177.0 & 0.18 & 3.2 & seasonal (1st harm.) \\
\hline LGO & $3.40 \mathrm{e}-02$ & 29.4 & 0.34 & 4.7 & circalunar & BRA & $9.89 \mathrm{e}-03$ & 101.1 & 0.19 & 3.3 & unknown \\
\hline \multirow[t]{2}{*}{ LGO } & $6.09 \mathrm{e}-02$ & 16.4 & 0.24 & 3.3 & & BRA & $3.39 \mathrm{e}-02$ & 29.5 & 0.66 & 11.7 & circalunar \\
\hline & & & & & & BRA & $6.07 \mathrm{e}-02$ & 16.5 & 0.17 & 3.1 & \\
\hline LSM & $0.00 \mathrm{e}+00$ & $\inf$ & 17.51 & 250.6 & mean & BRA & $6.78 \mathrm{e}-02$ & 14.8 & 0.30 & 5.3 & circalunar (1st harm.) \\
\hline LSM & $2.82 \mathrm{e}-03$ & 354.0 & 0.77 & 11.1 & seasonal & BRA & $7.06 \mathrm{e}-02$ & 14.2 & 0.19 & 3.3 & \\
\hline LSM & $5.65 \mathrm{e}-03$ & 177.0 & 0.24 & 3.4 & seasonal (1st harm.) & BRA & $7.77 \mathrm{e}-02$ & 12.9 & 0.19 & 3.3 & \\
\hline LSM & $9.89 \mathrm{e}-03$ & 101.1 & 0.24 & 3.5 & unknown & BRA & $1.03 \mathrm{e}-01$ & 9.7 & 0.17 & 3.0 & circalunar (2nd harm.) \\
\hline LSM & $3.39 \mathrm{e}-02$ & 29.5 & 0.33 & 4.8 & circalunar & & & & & & \\
\hline \multirow[t]{2}{*}{ LSM } & $6.07 \mathrm{e}-02$ & 16.5 & 0.24 & 3.4 & & FRE & $0.00 \mathrm{e}+00$ & $\inf$ & 18.60 & 267.6 & mean \\
\hline & & & & & & FRE & $2.82 \mathrm{e}-03$ & 354.0 & 0.76 & 10.9 & seasonal \\
\hline WEL & $0.00 \mathrm{e}+00$ & $\inf$ & 17.70 & 263.2 & mean & FRE & $3.39 \mathrm{e}-02$ & 29.5 & 0.71 & 10.2 & circalunar \\
\hline WEL & $2.82 \mathrm{e}-03$ & 354.0 & 0.86 & 12.8 & seasonal & FRE & $6.78 \mathrm{e}-02$ & 14.8 & 0.27 & 3.9 & circalunar (1st harm.) \\
\hline WEL & $5.65 \mathrm{e}-03$ & 177.0 & 0.22 & 3.3 & seasonal (1st harm.) & FRE & $7.06 \mathrm{e}-02$ & 14.2 & 0.23 & 3.3 & \\
\hline WEL & $9.89 \mathrm{e}-03$ & 101.1 & 0.21 & 3.1 & unknown & & & & & & \\
\hline WEL & $3.39 \mathrm{e}-02$ & 29.5 & 0.36 & 5.4 & circalunar & GRI & $0.00 \mathrm{e}+00$ & $\inf$ & 18.60 & 324.6 & mean \\
\hline \multirow{2}{*}{ WEL } & $6.07 \mathrm{e}-02$ & 16.5 & 0.21 & 3.1 & & GRI & $1.42 \mathrm{e}-03$ & 706.0 & 0.18 & 3.1 & \\
\hline & & & & & & GRI & $2.83 \mathrm{e}-03$ & 353.0 & 0.92 & 16.1 & seasonal \\
\hline LSW & $0.00 \mathrm{e}+00$ & $\inf$ & 17.73 & 256.2 & mean & GRI & $5.67 \mathrm{e}-03$ & 176.5 & 0.20 & 3.5 & seasonal (1st harm.) \\
\hline LSW & $2.82 \mathrm{e}-03$ & 354.0 & 0.58 & 8.4 & seasonal & GRI & $9.92 \mathrm{e}-03$ & 100.9 & 0.19 & 3.2 & unknown \\
\hline LSW & $9.89 \mathrm{e}-03$ & 101.1 & 0.24 & 3.5 & unknown & GRI & $2.69 \mathrm{e}-02$ & 37.2 & 0.17 & 3.0 & \\
\hline LSW & $3.39 \mathrm{e}-02$ & 29.5 & 0.41 & 6.0 & circalunar & GRI & $3.40 \mathrm{e}-02$ & 29.4 & 0.57 & 10.0 & circalunar \\
\hline LSW & $6.07 \mathrm{e}-02$ & 16.5 & 0.23 & 3.3 & & GRI & $6.09 \mathrm{e}-02$ & 16.4 & 0.18 & 3.2 & \\
\hline \multirow[t]{2}{*}{ LSW } & $7.06 \mathrm{e}-02$ & 14.2 & 0.21 & 3.1 & & GRI & $6.80 \mathrm{e}-02$ & 14.7 & 0.27 & 4.7 & circalunar (1st harm.) \\
\hline & & & & & & GRI & $1.03 \mathrm{e}-01$ & 9.7 & 0.17 & 3.0 & circalunar (2nd harm.) \\
\hline STW & $0.00 \mathrm{e}+00$ & $\inf$ & 17.93 & 259.4 & mean & & & & & & \\
\hline STW & $2.83 \mathrm{e}-03$ & 353.0 & 0.49 & 7.0 & seasonal & VOE & $0.00 \mathrm{e}+00$ & $\inf$ & 18.62 & 309.0 & mean \\
\hline STW & $3.40 \mathrm{e}-02$ & 29.4 & 0.45 & 6.5 & circalunar & VOE & $2.82 \mathrm{e}-03$ & 354.0 & 0.74 & 12.2 & seasonal \\
\hline STW & $6.09 \mathrm{e}-02$ & 16.4 & 0.23 & 3.3 & & VOE & $9.89 \mathrm{e}-03$ & 101.1 & 0.20 & 3.3 & unknown \\
\hline \multirow[t]{2}{*}{ STW } & $7.08 \mathrm{e}-02$ & 14.1 & 0.24 & 3.5 & & VOE & $2.12 \mathrm{e}-02$ & 47.2 & 0.18 & 3.0 & \\
\hline & & & & & & VOE & $3.39 \mathrm{e}-02$ & 29.5 & 0.64 & 10.6 & circalunar \\
\hline TRA & $0.00 \mathrm{e}+00$ & $\inf$ & 18.09 & 296.7 & mean & VOE & $6.78 \mathrm{e}-02$ & 14.8 & 0.32 & 5.3 & circalunar (1st harm.) \\
\hline TRA & $2.82 \mathrm{e}-03$ & 354.0 & 0.55 & 9.1 & seasonal & VOE & $7.06 \mathrm{e}-02$ & 14.2 & 0.23 & 3.8 & \\
\hline TRA & $3.39 \mathrm{e}-02$ & 29.5 & 0.46 & 7.5 & circalunar & VOE & $7.77 \mathrm{e}-02$ & 12.9 & 0.24 & 3.9 & \\
\hline TRA & $6.07 \mathrm{e}-02$ & 16.5 & 0.20 & 3.3 & & VOE & $1.03 \mathrm{e}-01$ & 9.7 & 0.18 & 3.0 & circalunar (2nd harm.) \\
\hline TRA & $6.78 \mathrm{e}-02$ & 14.8 & 0.21 & 3.4 & circalunar (1st harm.) & & & & & & \\
\hline \multirow[t]{2}{*}{ TRA } & $7.06 \mathrm{e}-02$ & 14.2 & 0.21 & 3.5 & & MAT & $0.00 \mathrm{e}+00$ & $\inf$ & 19.05 & 305.3 & mean \\
\hline & & & & & & MAT & $2.83 \mathrm{e}-03$ & 353.0 & 0.65 & 10.4 & seasonal \\
\hline STY & $0.00 \mathrm{e}+00$ & $\inf$ & 18.27 & 268.6 & mean & MAT & $9.92 \mathrm{e}-03$ & 100.9 & 0.25 & 3.9 & unknown \\
\hline STY & $2.82 \mathrm{e}-03$ & 354.0 & 0.69 & 10.1 & seasonal & MAT & $3.40 \mathrm{e}-02$ & 29.4 & 0.83 & 13.3 & circalunar \\
\hline STY & $9.89 \mathrm{e}-03$ & 101.1 & 0.23 & 3.3 & unknown & MAT & $6.52 \mathrm{e}-02$ & 15.3 & 0.24 & 3.9 & \\
\hline STY & $3.39 \mathrm{e}-02$ & 29.5 & 0.50 & 7.4 & circalunar & MAT & $6.80 \mathrm{e}-02$ & 14.7 & 0.32 & 5.1 & circalunar (1st harm.) \\
\hline STY & $6.07 \mathrm{e}-02$ & 16.5 & 0.21 & 3.1 & & MAT & $7.08 \mathrm{e}-02$ & 14.1 & 0.22 & 3.6 & \\
\hline STY & $6.78 \mathrm{e}-02$ & 14.8 & 0.23 & 3.5 & circalunar (1st harm.) & MAT & $7.79 \mathrm{e}-02$ & 12.8 & 0.23 & 3.8 & \\
\hline STY & $7.06 \mathrm{e}-02$ & 14.2 & 0.21 & 3.1 & & MAT & $1.78 \mathrm{e}-01$ & 5.6 & 0.19 & 3.1 & \\
\hline \multirow[t]{2}{*}{ STY } & $7.77 \mathrm{e}-02$ & 12.9 & 0.24 & 3.6 & & & & & & & \\
\hline & & & & & & MUN & $0.00 \mathrm{e}+00$ & $\inf$ & 19.76 & 421.4 & mean \\
\hline PAS & $0.00 \mathrm{e}+00$ & $\inf$ & 18.46 & 344.1 & mean & MUN & $2.82 \mathrm{e}-03$ & 354.0 & 0.56 & 12.0 & seasonal \\
\hline PAS & $2.82 \mathrm{e}-03$ & 354.0 & 0.57 & 10.7 & seasonal & MUN & $3.39 \mathrm{e}-02$ & 29.5 & 1.10 & 23.4 & circalunar \\
\hline PAS & $5.65 \mathrm{e}-03$ & 177.0 & 0.16 & 3.0 & seasonal (1st harm.) & MUN & $3.67 \mathrm{e}-02$ & 27.2 & 0.14 & 3.0 & \\
\hline PAS & $9.89 \mathrm{e}-03$ & 101.1 & 0.17 & 3.2 & unknown & MUN & $6.50 \mathrm{e}-02$ & 15.4 & 0.16 & 3.5 & \\
\hline PAS & $3.39 \mathrm{e}-02$ & 29.5 & 0.53 & 9.9 & circalunar & MUN & $6.78 \mathrm{e}-02$ & 14.8 & 0.45 & 9.6 & circalunar (1st harm.) \\
\hline PAS & $6.07 \mathrm{e}-02$ & 16.5 & 0.17 & 3.2 & & MUN & $7.06 \mathrm{e}-02$ & 14.2 & 0.24 & 5.1 & \\
\hline PAS & $6.50 \mathrm{e}-02$ & 15.4 & 0.17 & 3.1 & & MUN & $1.02 \mathrm{e}-01$ & 9.8 & 0.19 & 4.1 & circalunar (2nd harm.) \\
\hline PAS & $6.78 \mathrm{e}-02$ & 14.8 & 0.24 & 4.5 & circalunar (1st harm.) & & & & & & \\
\hline
\end{tabular}




\begin{tabular}{|c|c|c|c|c|c|c|c|c|c|c|c|}
\hline $\begin{array}{l}\text { COD } \\
(1)\end{array}$ & $\begin{array}{l}v \\
(2)\end{array}$ & $\begin{array}{l}\mathrm{T} \\
(3) \\
\end{array}$ & $\begin{array}{l}\text { A } \\
(4)\end{array}$ & $\begin{array}{l}\mathrm{S} / \mathrm{N} \\
(5)\end{array}$ & $\begin{array}{l}\text { note } \\
(6)\end{array}$ & $\begin{array}{l}\text { COD } \\
(1)\end{array}$ & $\begin{array}{l}v \\
(2)\end{array}$ & $\begin{array}{l}\mathrm{T} \\
(3) \\
\end{array}$ & $\begin{array}{l}\text { A } \\
(4)\end{array}$ & $\begin{array}{l}\mathrm{S} / \mathrm{N} \\
(5)\end{array}$ & $\begin{array}{l}\text { note } \\
(6)\end{array}$ \\
\hline GIS & $0.00 \mathrm{e}+00$ & inf & 20.07 & 400.5 & mean & FEU & $0.00 \mathrm{e}+00$ & inf & 20.66 & 362.6 & mean \\
\hline GIS & $1.42 \mathrm{e}-03$ & 706.0 & 0.21 & 4.2 & & FEU & $3.11 \mathrm{e}-02$ & 32.2 & 0.20 & 3.5 & \\
\hline GIS & $5.67 \mathrm{e}-03$ & 176.5 & 0.18 & 3.6 & seasonal (1st harm.) & FEU & $3.39 \mathrm{e}-02$ & 29.5 & 1.22 & 21.5 & circalunar \\
\hline GIS & $7.08 \mathrm{e}-03$ & 141.2 & 0.21 & 4.1 & & FEU & $3.67 \mathrm{e}-02$ & 27.2 & 0.19 & 3.4 & \\
\hline GIS & $8.50 \mathrm{e}-03$ & 117.7 & 0.23 & 4.7 & & FEU & $6.78 \mathrm{e}-02$ & 14.8 & 0.60 & 10.5 & circalunar (1st harm.) \\
\hline GIS & $1.13 \mathrm{e}-02$ & 88.2 & 0.16 & 3.3 & & FEU & $7.06 \mathrm{e}-02$ & 14.2 & 0.23 & 4.0 & \\
\hline GIS & $1.27 \mathrm{e}-02$ & 78.4 & 0.18 & 3.7 & & FEU & $1.02 \mathrm{e}-01$ & 9.8 & 0.24 & 4.2 & circalunar (2nd harm.) \\
\hline GIS & $1.42 \mathrm{e}-02$ & 70.6 & 0.18 & 3.6 & & FEU & $1.24 \mathrm{e}-01$ & 8.0 & 0.19 & 3.3 & \\
\hline GIS & $1.56 \mathrm{e}-02$ & 64.2 & 0.18 & 3.7 & & & & & & & \\
\hline GIS & $1.84 \mathrm{e}-02$ & 54.3 & 0.17 & 3.3 & & KRI & $0.00 \mathrm{e}+00$ & $\inf$ & 20.81 & 428.6 & mean \\
\hline GIS & $2.55 \mathrm{e}-02$ & 39.2 & 0.18 & 3.6 & & KRI & $3.12 \mathrm{e}-02$ & 32.1 & 0.28 & 5.8 & \\
\hline GIS & $3.12 \mathrm{e}-02$ & 32.1 & 0.18 & 3.6 & & KRI & $3.40 \mathrm{e}-02$ & 29.4 & 1.16 & 23.9 & circalunar \\
\hline GIS & $3.40 \mathrm{e}-02$ & 29.4 & 1.02 & 20.3 & circalunar & KRI & $6.09 \mathrm{e}-02$ & 16.4 & 0.16 & 3.2 & \\
\hline GIS & $6.80 \mathrm{e}-02$ & 14.7 & 0.47 & 9.4 & circalunar (1st harm.) & KRI & $6.80 \mathrm{e}-02$ & 14.7 & 0.51 & 10.6 & circalunar (1st harm.) \\
\hline GIS & $7.08 \mathrm{e}-02$ & 14.1 & 0.30 & 6.0 & & KRI & $7.08 \mathrm{e}-02$ & 14.1 & 0.16 & 3.2 & \\
\hline \multirow[t]{2}{*}{ GIS } & $1.02 \mathrm{e}-01$ & 9.8 & 0.19 & 3.7 & circalunar (2nd harm.) & KRI & $9.49 \mathrm{e}-02$ & 10.5 & 0.15 & 3.1 & \\
\hline & & & & & & KRI & $1.02 \mathrm{e}-01$ & 9.8 & 0.19 & 3.9 & circalunar (2nd harm.) \\
\hline ULI & $0.00 \mathrm{e}+00$ & $\inf$ & 20.13 & 353.8 & mean & KRI & $1.29 \mathrm{e}-01$ & 7.8 & 0.16 & 3.3 & \\
\hline ULI & $2.83 \mathrm{e}-03$ & 353.0 & 0.50 & 8.8 & seasonal & & & & & & \\
\hline ULI & $8.50 \mathrm{e}-03$ & 117.7 & 0.19 & 3.3 & & LOS & $0.00 \mathrm{e}+00$ & $\inf$ & 20.86 & 355.1 & mean \\
\hline ULI & $1.13 \mathrm{e}-02$ & 88.2 & 0.18 & 3.1 & & LOS & $1.27 \mathrm{e}-02$ & 78.7 & 0.20 & 3.4 & \\
\hline ULI & $1.56 \mathrm{e}-02$ & 64.2 & 0.21 & 3.7 & & LOS & $2.40 \mathrm{e}-02$ & 41.6 & 0.21 & 3.5 & \\
\hline ULI & $2.12 \mathrm{e}-02$ & 47.1 & 0.25 & 4.3 & & LOS & $3.11 \mathrm{e}-02$ & 32.2 & 0.24 & 4.0 & \\
\hline ULI & $2.83 \mathrm{e}-02$ & 35.3 & 0.18 & 3.1 & & LOS & $3.39 \mathrm{e}-02$ & 29.5 & 1.30 & 22.0 & circalunar \\
\hline ULI & $3.12 \mathrm{e}-02$ & 32.1 & 0.29 & 5.2 & & LOS & $3.67 \mathrm{e}-02$ & 27.2 & 0.20 & 3.5 & \\
\hline ULI & $3.40 \mathrm{e}-02$ & 29.4 & 1.16 & 20.4 & circalunar & LOS & $6.78 \mathrm{e}-02$ & 14.8 & 0.56 & 9.5 & circalunar (1st harm.) \\
\hline ULI & $3.97 \mathrm{e}-02$ & 25.2 & 0.21 & 3.8 & & LOS & $7.06 \mathrm{e}-02$ & 14.2 & 0.31 & 5.2 & \\
\hline ULI & $6.52 \mathrm{e}-02$ & 15.3 & 0.19 & 3.4 & & & & & & & \\
\hline ULI & $6.66 \mathrm{e}-02$ & 15.0 & 0.18 & 3.1 & & $\mathrm{ZOE}$ & $0.00 \mathrm{e}+00$ & $\inf$ & 21.01 & 378.2 & mean \\
\hline ULI & $6.80 \mathrm{e}-02$ & 14.7 & 0.45 & 7.9 & circalunar (1st harm.) & $\mathrm{ZOE}$ & $1.55 \mathrm{e}-02$ & 64.4 & 0.17 & 3.1 & \\
\hline \multirow[t]{2}{*}{ ULI } & $7.08 \mathrm{e}-02$ & 14.1 & 0.20 & 3.5 & & $\mathrm{ZOE}$ & $1.98 \mathrm{e}-02$ & 50.6 & 0.25 & 4.5 & \\
\hline & & & & & & $\mathrm{ZOE}$ & $3.39 \mathrm{e}-02$ & 29.5 & 1.55 & 28.0 & circalunar \\
\hline KID & $0.00 \mathrm{e}+00$ & $\inf$ & 20.37 & 506.3 & mean & $\mathrm{ZOE}$ & $3.67 \mathrm{e}-02$ & 27.2 & 0.39 & 7.1 & \\
\hline KID & $7.06 \mathrm{e}-03$ & 141.6 & 0.14 & 3.6 & & $\mathrm{ZOE}$ & $3.81 \mathrm{e}-02$ & 26.2 & 0.20 & 3.7 & \\
\hline KID & $9.89 \mathrm{e}-03$ & 101.1 & 0.14 & 3.5 & unknown & $\mathrm{ZOE}$ & $3.95 \mathrm{e}-02$ & 25.3 & 0.19 & 3.5 & \\
\hline KID & $1.27 \mathrm{e}-02$ & 78.7 & 0.14 & 3.5 & & $\mathrm{ZOE}$ & $6.78 \mathrm{e}-02$ & 14.8 & 0.62 & 11.2 & circalunar (1st harm.) \\
\hline KID & $1.55 \mathrm{e}-02$ & 64.4 & 0.14 & 3.4 & & $\mathrm{ZOE}$ & $7.06 \mathrm{e}-02$ & 14.2 & 0.28 & 5.1 & \\
\hline KID & $1.84 \mathrm{e}-02$ & 54.5 & 0.13 & 3.3 & & & & & & & \\
\hline KID & $2.54 \mathrm{e}-02$ & 39.3 & 0.17 & 4.3 & & BOD & $0.00 \mathrm{e}+00$ & $\inf$ & 21.04 & 329.6 & mean \\
\hline KID & $3.11 \mathrm{e}-02$ & 32.2 & 0.14 & 3.4 & & BOD & $5.62 \mathrm{e}-03$ & 178.0 & 0.20 & 3.1 & \\
\hline KID & $3.39 \mathrm{e}-02$ & 29.5 & 1.19 & 29.6 & circalunar & BOD & $9.36 \mathrm{e}-03$ & 106.8 & 0.23 & 3.5 & \\
\hline KID & $3.67 \mathrm{e}-02$ & 27.2 & 0.20 & 5.0 & & BOD & $2.81 \mathrm{e}-02$ & 35.6 & 0.20 & 3.1 & \\
\hline KID & $6.50 \mathrm{e}-02$ & 15.4 & 0.12 & 3.1 & & BOD & $3.18 \mathrm{e}-02$ & 31.4 & 0.23 & 3.6 & \\
\hline KID & $6.78 \mathrm{e}-02$ & 14.8 & 0.52 & 13.0 & circalunar (1st harm.) & BOD & $3.37 \mathrm{e}-02$ & 29.7 & 1.51 & 23.6 & circalunar \\
\hline KID & $7.06 \mathrm{e}-02$ & 14.2 & 0.25 & 6.3 & & BOD & $3.56 \mathrm{e}-02$ & 28.1 & 0.48 & 7.6 & \\
\hline KID & $1.02 \mathrm{e}-01$ & 9.8 & 0.20 & 4.9 & circalunar (2nd harm.) & BOD & $3.93 \mathrm{e}-02$ & 25.4 & 0.34 & 5.3 & \\
\hline \multirow[t]{2}{*}{ KID } & $1.34 \mathrm{e}-01$ & 7.5 & 0.12 & 3.0 & circalunar (3rd harm.) & BOD & $6.74 \mathrm{e}-02$ & 14.8 & 0.56 & 8.7 & circalunar (1st harm.) \\
\hline & & & & & & BOD & $6.93 \mathrm{e}-02$ & 14.4 & 0.29 & 4.5 & \\
\hline GRU & $0.00 \mathrm{e}+00$ & $\inf$ & 20.39 & 568.6 & mean & BOD & $7.49 \mathrm{e}-02$ & 13.3 & 0.20 & 3.1 & \\
\hline GRU & $2.83 \mathrm{e}-03$ & 353.0 & 0.38 & 10.6 & seasonal & BOD & $9.74 \mathrm{e}-02$ & 10.3 & 0.23 & 3.6 & \\
\hline GRU & $3.12 \mathrm{e}-02$ & 32.1 & 0.17 & 4.6 & & BOD & $1.01 \mathrm{e}-01$ & 9.9 & 0.20 & 3.1 & circalunar (2nd harm.) \\
\hline GRU & $3.26 \mathrm{e}-02$ & 30.7 & 0.13 & 3.6 & & & & & & & \\
\hline GRU & $3.40 \mathrm{e}-02$ & 29.4 & 1.34 & 37.3 & circalunar & & & & & & \\
\hline GRU & $3.68 \mathrm{e}-02$ & 27.2 & 0.28 & 7.7 & & & & & & & \\
\hline GRU & $6.52 \mathrm{e}-02$ & 15.3 & 0.19 & 5.3 & & & & & & & \\
\hline GRU & $6.80 \mathrm{e}-02$ & 14.7 & 0.53 & 14.8 & circalunar (1st harm.) & & & & & & \\
\hline GRU & $7.08 \mathrm{e}-02$ & 14.1 & 0.23 & 6.4 & & & & & & & \\
\hline GRU & $1.02 \mathrm{e}-01$ & 9.8 & 0.15 & 4.1 & circalunar (2nd harm.) & & & & & & \\
\hline GRU & $1.05 \mathrm{e}-01$ & 9.5 & 0.11 & 3.1 & & & & & & & \\
\hline
\end{tabular}




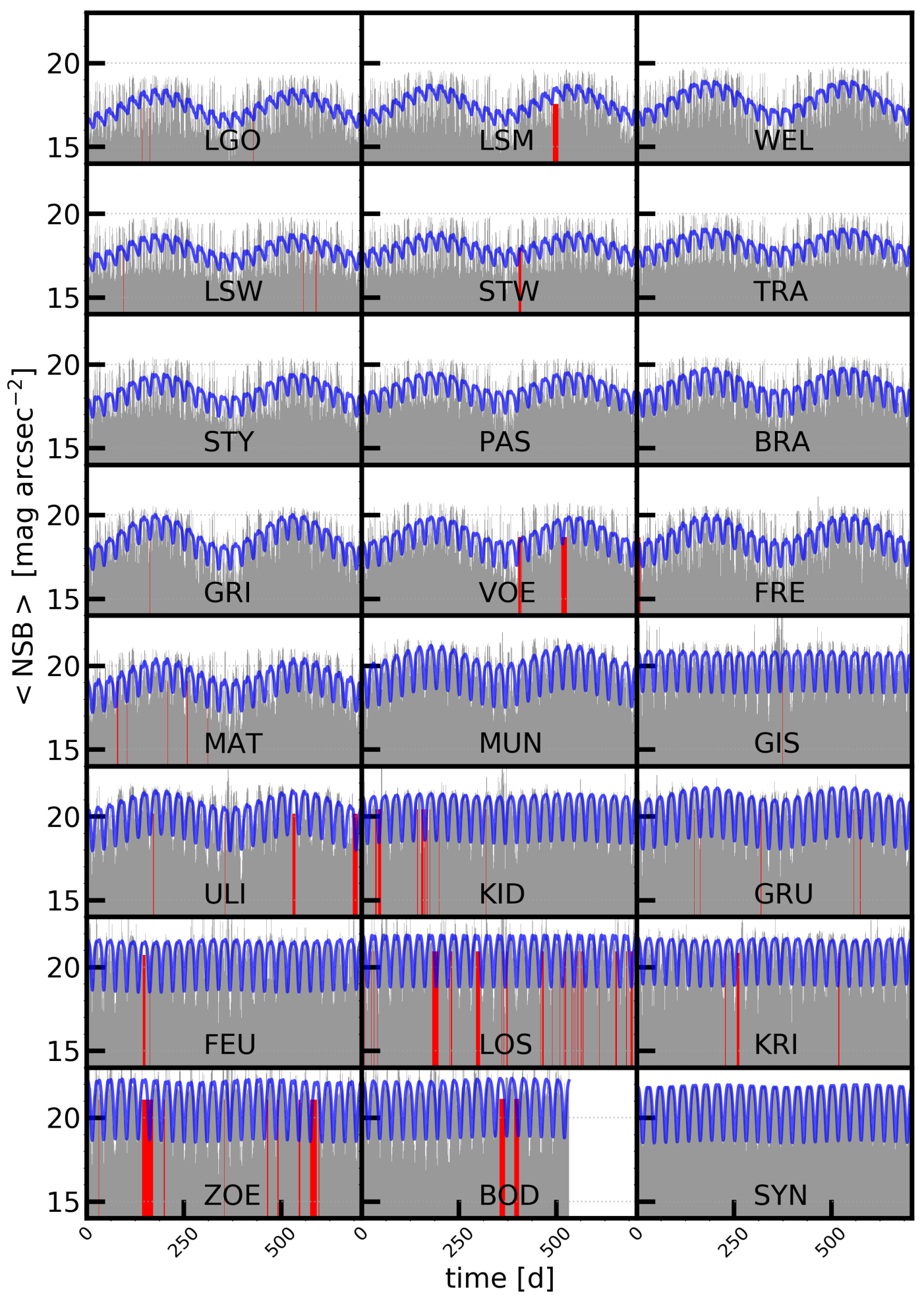

Figure 12. The gray shaded areas in the panels show $<\mathrm{NSB}>$ time series for 2016 and 2017 obtained from 23 SQM stations in Upper Austria and from a cloud-free synthetic sky model (SYN) as described in section 3.2. On top of that, the result of an inverse FFT (iFFT) of identified frequency components is shown as blue line. The iFFT frequencies correspond to the mean brightness level, the circalunar rhythm plus its first two harmonics as well as a yearly cycle, i.e. bright winters and dark summers. This cycle is driven by an increase of overcast skies during the winter months and an amplification of light pollution by clouds. Data gaps that were set to the mean $<\mathrm{NSB}>$ value are marked in red. 


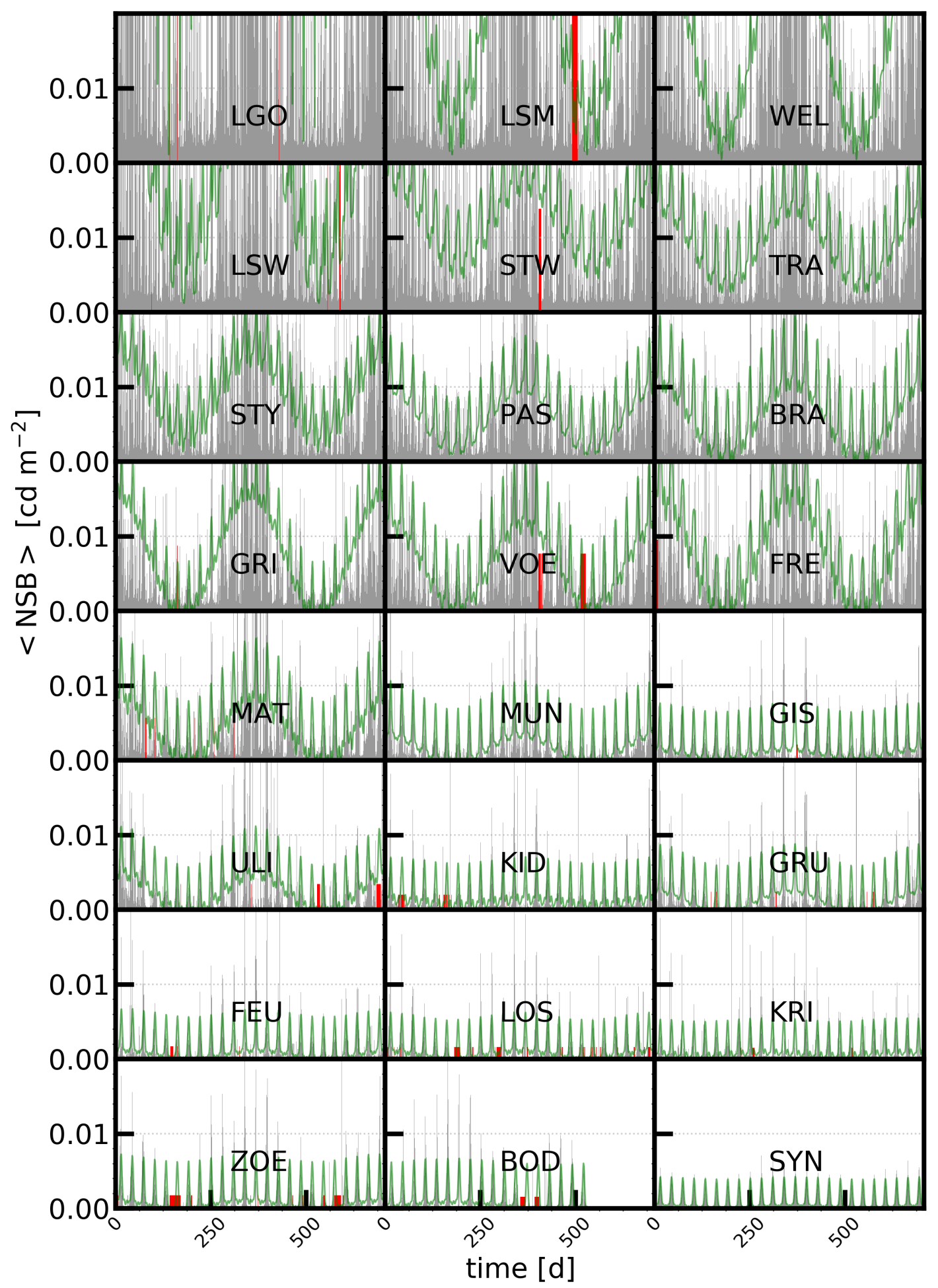

Figure 13. The gray shaded areas in the panels show $<\mathrm{NSB}>$ time series for 2016 and 2017 obtained from 23 SQM stations in Upper Austria and from a cloud-free synthetic sky model (SYN) as described in section 3.2. On top of that, the result of an inverse FFT (iFFT) of identified frequency components is shown as blue line. The iFFT frequencies correspond to the mean brightness level, the circalunar rhythm plus its first two harmonics as well as a yearly cycle, i.e. bright winters and dark summers. This cycle is driven by an increase of overcast skies during the winter months and an amplification of light pollution by clouds. Data gaps that were set to the mean $\langle$ NSB $>$ value are marked in red. 